\title{
Article \\ Generalized Mittag-Leffler Kernel Form Solutions of Free Convection Heat and Mass Transfer Flow of Maxwell Fluid with Newtonian Heating: Prabhakar Fractional Derivative Approach
}

\author{
Aziz Ur Rehman ${ }^{1}$, Fahd Jarad ${ }^{2,3,4}$ (D), Muhammad Bilal Riaz ${ }^{1,5,6, * \mathbb{D}}$ and Zaheer Hussain Shah ${ }^{7}$ \\ 1 Department of Mathematics, University of Management and Technology, Lahore 54770, Pakistan; \\ prof.azizkhan@gmail.com \\ 2 Department of Mathematics, Cankaya University, Ankara 06790, Turkey; fahd@cankaya.edu.tr \\ 3 Department of Mathematics, King Abdulaziz University, Jeddah 21589, Saudi Arabia \\ 4 Department of Medical Research, China Medical University, Taichung 40402, Taiwan \\ 5 Department of Automation, Biomechanics and Mechatronics, Lodz University of Technology, \\ 1/15 Stefanowskiego St., 90-924 Lodz, Poland \\ 6 Institute for Groundwater Studies, University of the Free State, Bloemfontein 9301, South Africa \\ 7 Department of Physics, University of Management and Technology, Lahore 54770, Pakistan; \\ zaheer.hussain@umt.edu.pk \\ * Correspondence: Muhammad.riaz@p.lodz.pl
}

check for

updates

Citation: Rehman, A.U.; Jarad, F.; Riaz, M.B.; Shah, Z.H. Generalized Mittag-Leffler Kernel Form Solutions of Free Convection Heat and Mass Transfer Flow of Maxwell Fluid with Newtonian Heating: Prabhakar Fractional Derivative Approach. Fractal Fract. 2022, 6, 98. https:// doi.org/10.3390/fractalfract6020098

Academic Editor: Wojciech Sumelka

Received: 3 January 2022

Accepted: 3 February 2022

Published: 10 February 2022

Publisher's Note: MDPI stays neutral with regard to jurisdictional claims in published maps and institutional affiliations.

Copyright: (C) 2022 by the authors. Licensee MDPI, Basel, Switzerland. This article is an open access article distributed under the terms and conditions of the Creative Commons Attribution (CC BY) license (https:// creativecommons.org/licenses/by/ $4.0 /)$.

\begin{abstract}
In this article, the effects of Newtonian heating along with wall slip condition on temperature is critically examined on unsteady magnetohydrodynamic (MHD) flows of Prabhakar-like non integer Maxwell fluid near an infinitely vertical plate under constant concentration. For the sake of generalized memory effects, a new mathematical fractional model is formulated based on a newly introduced Prabhakar fractional operator with generalized Fourier's law and Fick's law. This fractional model has been solved analytically and exact solutions for dimensionless velocity, concentration, and energy equations are calculated in terms of Mittag-Leffler functions by employing the Laplace transformation method. Physical impacts of different parameters such as $\alpha, P r, \beta, S c, G r$, $\gamma$, and $G m$ are studied and demonstrated graphically by Mathcad software. Furthermore, to validate our current results, some limiting models such as classical Maxwell model, classical Newtonian model, and fractional Newtonian model are recovered from Prabhakar fractional Maxwell fluid. Moreover, we compare the results between Maxwell and Newtonian fluids for both fractional and classical cases with and without slip conditions, showing that the movement of the Maxwell fluid is faster than viscous fluid. Additionally, it is visualized that both classical Maxwell and viscous fluid have relatively higher velocity as compared to fractional Maxwell and viscous fluid.
\end{abstract}

Keywords: Prabhakar derivative; magnetic effect; slip conditions; analytical solution; Mittag-Leffler functions; physical aspect via graphs

\section{Introduction}

It is a well-known fact that many scientists and researchers have more interest in exploring non-Newtonian fluids due to their wide practical applications in modern technologies and significant characteristics. The properties of non-Newtonian fluids are demonstrated in various industrial sectors because they play a vital role in manufacturing, e.g., greases, clay coatings, polymer melts, waste liquid, extrusion of molten plastic, pharmaceutical, polymer processing, oil and gas, well drilling, food processing industries, and many emulsions. For instance, shampoo, drilling mud, biological materials, polymer melts, all emulsions, and complex mixtures are considered as non-Newtonian fluids. The non-Newtonian fluids have different characteristics and can not be described in a single model, but in the case of Newtonian fluid it is possible to express in a single model. It is quite ambiguous how to classify non-Newtonian fluids because, in the literature, several types of fluid exist. 
However, non-Newtonian fluids are classified into three types, rate, differential, and integral. Researchers studied these three types of non-Newtonian models, and each model has different characteristics. Some common models that describe the computational and physical characteristics of non-Newtonian fluids are second grade and third grade models, the Jeffery model, Casson model, Maxwell model, and power law model [1-6]. Such fluid models are simple, but each model has certain limitations; for example, second grade fluid is a simple sub-class of a differential type of non-Newtonian fluid. Many scientists and researchers are interested to explore the geometry of the flow regime of second grade fluid and have discussed many interesting features in different configurations [7-13]. However, the second grade fluid model does not provide sufficient knowledge about viscosity, only describing the effects of elasticity. Identically, the power law model efficiently explains the fluids viscosity, but is unable to provide information regarding effects of elasticity. Further, these fluid models do not incorporate the relaxation time. Flow analysis of such fluids have great importance for practical and theoretical studies in many industrial sectors. Among them, the Maxwell fluid model, which is a simple sub-division of the rate type of non-Newtonian fluids for authentic approximation of this phenomenon, has attracted special attention. Maxwell fluid has both properties (viscosity and elasticity), so it is named as viscoelastic fluid. The Maxwell fluid model was initially proposed by James Clerk Maxwell in 1867. The Maxwell model was developed with an aim to foretell the elastic and viscous behavior of air [14]. However, some researchers frequently operated the Maxwell model in response to different viscoelastic fluids ranging from polymeric fluids to the Earth's mantle. Olsson et al. [15] discussed some rheological characteristics of Maxwell fluid. Aman et al. [16] studied generalized Maxwell migration in a porous media under second order slip effects. Choi et al. [17] discussed Maxwell fluid flow behavior passing through a channel. The computational characteristics of viscosity variation of Maxwell fluid flow through a thick surface with thermal stratifications was presented by Khan et al. [18]. Fetecau et al. $[19,20]$ explored the Maxwell fluid models and obtained new analytical solutions. Hosseinzadeh et al. [21] analyzed the effect of Joule heating and heat generation on chemically reactive motion of Maxwell fluid by employing two dimensional form of the Darcy-Forchheimer relation. Riaz et al. [22,23] described interesting facts regarding Maxwell fluid, and numerical solutions with stability analysis using different fractional operators are studied in [24-27].

Nowadays, the branch of mathematics fractional order calculus has been growing immensely on account of its enormous significance in science and engineering that are absent in non-fractional calculus, which deals with an arbitrary order of integration and differentiation. Fractional differential equations are massively applied to model various daily life physical problems because fractional calculus has memory effects, such as problems in fluid flow, diffusion, relaxation, reaction, oscillation, dynamical processes, and retardation processes in complex systems and many more engineering processes, wherefore ordinary models can not anticipate the preceding processes state. In the literature, most of the studies are focused on flow problems relative to several fractional operators with local kernels, as well as non-local kernels such as Marchaud-Caputo, Atangana-Baleanu, Caputo-Fabrizio, Prabhakar fractional derivative, and others [28-30]. These indicate the current state but also the future state of a system. Yavuz et al. [31] applied Liouville-Caputo fractional derivative with its generalized version to solve the fractional incompressible second-grade fluid differential equations by combining both the $\rho$-Laplace homotopy transform method ( $\rho$-LHTM) and the heat balance integral method (HBIM) successfully. A linear visco-elastic model with the application of a Prabhakar fractional operator has been investigated by Giusti and Colombaro [32]. Further, comparative study for fractional model of MHD Maxwell fluid to anticipate the heat impacts was established by Riaz et al. [33]. Ozkose et al. [34,35] developed a fractional model of tumor-immune system interaction related to lung cancer and also studied the interactions between COVID-19 and diabetes with hereditary traits using real data. Naik et al. [36] analyzed COVID-19 epidemics with treatment in fractional derivatives on the base of data from Pakistan and some other studies regarding COVID-19 
epidemic model investigated by Ikram et al. [37], Allegretti et al. [38], and Joshi et al. [39]. Furthermore, some respective studies associated with fractionalized models are discussed in detail; see, for instance, [40-43]; most of the studies are focused on flow problems by considering different fluids, related to fractional operators and heat transport phenomena.

Xiao-Hong Zhang et al. [44] recently, investigated the flow of a generalized fractional Prabhakar-type Maxwell fluid model, without analyzing the impacts of diffusion equation, and the results obtained via application of a Laplace transformation from the proposed problem. In the considered model, a new approach was used to fractionalize the diffusion equation by applying the definition of the Prabhakar fractional operator along with the generalized Fick's law; the influence of fractionalized diffusion equation is analyzed on momentum equation. Based on the above mentioned discussion, the prominent features of this derivation is to construct a new mathematical fractional model based on the newly introduced Prabhakar fractional operator with generalized Fourier's law and Fick's law. This fractional model has been solved analytically, and exact solutions for dimensionless velocity, concentration, and energy equations are calculated in terms of Mittag-Leffler functions by employing the Laplace transformation method. Physical impacts of different parameters such as $\alpha, \operatorname{Pr}, \beta, S c, G r, \gamma$, and $G m$ are studied and demonstrated graphically by Mathcad software. Furthermore, to validate our current results, some limiting models such as the classical Maxwell model, classical Newtonian model, and fractional Newtonian model are recovered from the Prabhakar fractional Maxwell model.

\section{Mathematical Model}

Consider the time dependent, incompressible, electrically conducting natural convective movement of Maxwell fluid over an erected plate which is also non conductive having infinite length, along with wall slip condition on temperature. Initially, suppose that, at time $\eta=0$, the fluid and plate both are static having fixed species concentration $C_{\infty}$ and the ambient temperature $T_{\infty}$. For time $\eta=0^{+}$, the plate is still at rest, while the temperature is stabilized in the form $T(0, \eta)-\omega \frac{\partial T(0, \eta)}{\partial \phi}=u_{0} f(\eta)$, whereas concentration is maintained at the value $C_{w}$ and geometry of the proposed problem is configured in Figure 1 . In the present work, the fluid velocity, temperature, and concentration are functions of $\phi$ and time $\eta$ only, because the plate is infinite due to which the fluid properties only depend on $\phi$ and time $\eta$; therefore, velocity field, temperature, and concentration take the form of $\vec{U}(\phi, \eta)=u(\phi, \eta) \hat{i}, T(\phi, \eta)$, and $C(\phi, \eta)$, respectively, where $\hat{i}$ represents the unit vector in the $\mathrm{x}$ direction and $u(\phi, \eta)$ is the $\mathrm{x}$-component of the velocity. Further, the fluid velocity satisfies the continuity equation in the presence of these factors.

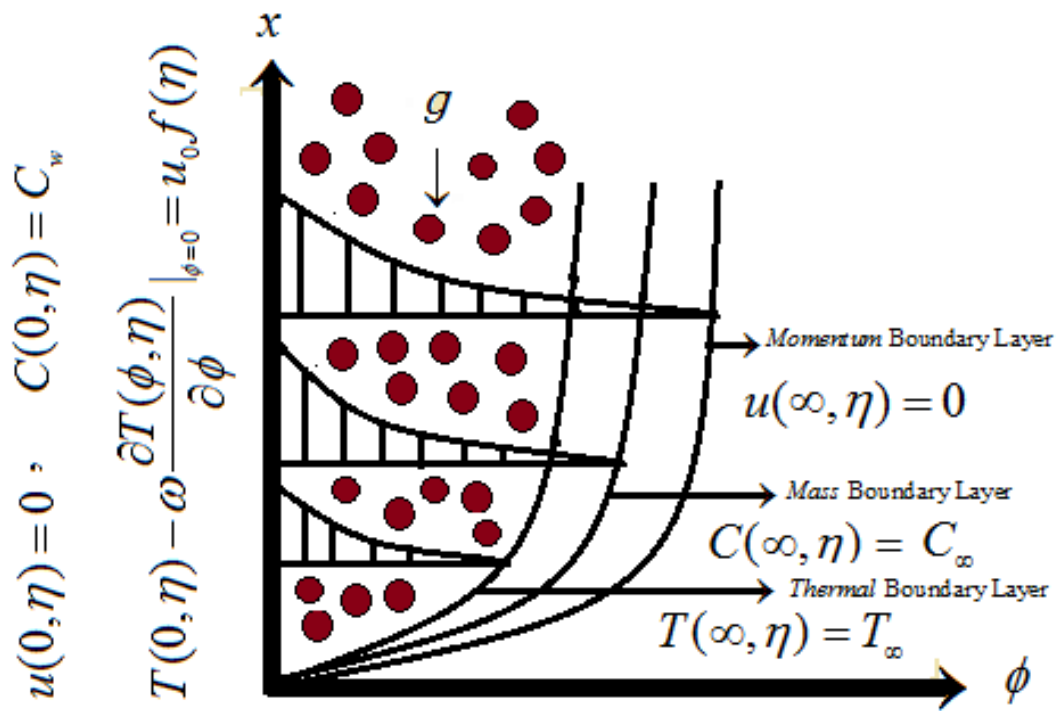

Figure 1. Geometrical formation of the flow model. 
The movement of the fluid and thermal transport govern partial differential equations of the considered problem for MHD Maxwell fluid under Boussinesq's approximation [45].

The momentum equation

$$
\left(1+\lambda \frac{\partial}{\partial \eta}\right) \frac{\partial u(\phi, \eta)}{\partial \eta}=v \frac{\partial^{2} u(\phi, \eta)}{\partial \phi^{2}}+\left(1+\lambda \frac{\partial}{\partial \eta}\right)\left[g \beta_{T}\left(T(\phi, \eta)-T_{\infty}\right)+g_{C}\left(C(\phi, \eta)-C_{\infty}\right)\right]
$$

The energy balance equation

$$
C_{p} \frac{\partial T(\phi, \eta)}{\partial \eta}=-\frac{1}{\rho} \frac{\partial q(\phi, \eta)}{\partial \phi} .
$$

Fourier's thermal flux law

$$
q(\phi, \eta)=-k \frac{\partial T(\phi, \eta)}{\partial \phi}
$$

The diffusion equation

$$
\frac{\partial C(\phi, \eta)}{\partial \eta}=-\frac{\partial \chi(\phi, \eta)}{\partial \phi}
$$

Fick's Law

$$
\chi(\phi, \eta)=-D_{m} \frac{\partial C(\phi, \eta)}{\partial \phi} .
$$

with associated initial/boundary conditions

$$
\begin{aligned}
& u(\phi, 0)=0, \quad T(\phi, 0)=T_{\infty}, \quad C(\phi, 0)=C_{\infty}, \quad \phi \geq 0, \\
& u(0, \eta)=0, \quad T(0, \eta)-\left.\omega \frac{\partial T(\phi, \eta)}{\partial \phi}\right|_{\phi=0}=u_{0} f(\eta), \quad C(0, \eta)=C_{w}, \quad \eta \geq 0, \\
& u(\phi, \eta) \rightarrow 0, \quad T(\phi, \eta) \rightarrow \infty, \quad C(\phi, \eta) \rightarrow \infty \quad \text { as } \quad \phi \rightarrow \infty .
\end{aligned}
$$

To obtain the non-dimentionalized equations, the following new on-dimensional quantities are introduced:

$$
\begin{aligned}
& \eta^{*}=\frac{u_{0}^{2} \eta}{v}, \quad \phi^{*}=\frac{u_{0} \phi}{v}, \quad u^{*}=\frac{u}{u_{0}}, \quad v=\frac{\mu}{\rho}, \quad T^{*}=\frac{T-T_{\infty}}{T_{w}-T_{\infty}}, \quad C^{*}=\frac{C-C_{\infty}}{C_{w}-C_{\infty}}, \\
& \lambda^{*}=\frac{u_{0}^{2} \lambda}{v}, \quad q^{*}=\frac{q}{q_{0}}, \quad \chi^{*}=\frac{\chi}{\chi_{0}}, \quad q_{0}=\frac{k\left(T_{w}-T_{\infty}\right) u_{0}}{v}, \quad \chi_{0}=\frac{D_{m}\left(C_{w}-C_{\infty}\right) u_{0}}{v}, \\
& G r=\frac{g \beta_{T}\left(T_{w}-T_{\infty}\right)}{u_{0}^{3}}, \quad G m=\frac{g \beta_{C}\left(C_{w}-C_{\infty}\right)}{u_{0}^{3}}, \quad \operatorname{Pr}=\frac{\mu C_{p}}{k}, \quad S c=\frac{v}{D_{m}} .
\end{aligned}
$$

After substituting Equation (7) into Equations (1)-(5) and ignoring the notation of asterisk $*$, we find all equations in dimentionless form, as:

$$
\begin{gathered}
\left(1+\lambda \frac{\partial}{\partial \eta}\right) \frac{\partial u(\phi, \eta)}{\partial \eta}=\frac{\partial^{2} u(\phi, \eta)}{\partial \phi^{2}}+\left(1+\lambda \frac{\partial}{\partial \eta}\right) \operatorname{Gr} T(\phi, \eta)+\left(1+\lambda \frac{\partial}{\partial \eta}\right) G m C(\phi, \eta) \\
\frac{\partial T(\phi, \eta)}{\partial \eta}=-\frac{1}{\operatorname{Pr}} \frac{\partial q(\phi, \eta)}{\partial \phi} \\
q(\phi, \eta)=-\frac{\partial T(\phi, \eta)}{\partial \phi}
\end{gathered}
$$




$$
\begin{gathered}
\frac{\partial C(\phi, \eta)}{\partial \eta}=-\frac{1}{S c} \frac{\partial \chi(\phi, \eta)}{\partial \phi} \\
\chi(\phi, \eta)=-\frac{\partial C(\phi, \eta)}{\partial \phi} .
\end{gathered}
$$

Additionally, the set of initial and boundary conditions in non-dimensional form are stated as:

$$
\begin{gathered}
u(\phi, 0)=0, \quad T(\phi, 0)=0, \quad C(\phi, 0)=0, \quad \text { for } \phi \geq 0, \\
u(0, \eta)=0, \quad T(0, \eta)-\left.\omega \frac{\partial T(\phi, \eta)}{\partial \phi}\right|_{\phi=0}=f(\eta), \quad C(0, \eta)=1, \quad \text { for } \eta \geq 0, \\
u(\phi, \eta) \rightarrow 0, \quad T(\phi, \eta) \rightarrow 0, \quad C(\phi, \eta) \rightarrow 0 \quad \text { as } \phi \rightarrow \infty .
\end{gathered}
$$

\section{Preliminaries}

The regularized Prabhakar derivative is described as:

$$
{ }^{C} D_{\alpha, \beta, \wp}^{\gamma} f(t)=E_{\alpha, m-\beta, \wp}^{-\gamma} f^{(m)}(t)=\int_{0}^{t}(t-\tau)^{m-\beta-1} E_{\alpha, m-\beta}^{-\gamma}\left(\wp(t-\tau)^{\alpha}\right) f^{(m)}(\tau) d \tau .
$$

where

$$
E_{\alpha, \beta, \wp}^{\gamma} f(t)=\int_{0}^{t}(t-\tau)^{\beta-1} E_{\alpha, \beta}^{\gamma}\left(\wp(t-\tau)^{\alpha}\right) f(\tau) d \tau .
$$

represents the Prabhakar integral, and

$$
E_{\alpha, \beta}^{\gamma}(z)=\sum_{n=0}^{\infty} \frac{\Gamma(\gamma+n) z^{n}}{n ! \Gamma(\gamma) \Gamma(\alpha n+\beta)}, \quad \alpha, \beta, \gamma, z \in \mathbb{C}, \quad \operatorname{Re}(\alpha)>0
$$

is the three-parameter Mittag-Leffler function. Additionally, the function $t^{\beta-1} E_{\alpha, \beta}^{\gamma}\left(\wp t^{\alpha}\right)$ with $t \in \mathbb{R}, \alpha, \beta, \gamma, \wp \in \mathbb{C}, \operatorname{Re}(\alpha)>0$ is called the Prabhakar kernel.

The Laplace transformation of the regularized Prabhakar derivative is described as:

$$
\mathscr{L}\left\{{ }^{C} D_{\alpha, \beta, \wp}^{\gamma} f(t)\right\}=\xi^{\beta-m}\left(1-\wp \xi^{-\alpha}\right)^{\gamma} \mathscr{L}\left\{f^{(m)}(t)\right\}
$$

where $\alpha, \beta$, and $\gamma$ represent the fractional parameters and $\xi$ denoted by Laplace transform parameter.

\section{Solution of the Problem}

In the present study, we introduce a novel mathematical model named Prabhakar's fractional operator which generalizes the thermal memory effects. The generalized Fourier and Fick's laws are based on Prabhakar's fractional derivative, and are defined as:

$$
\begin{aligned}
& q(\phi, \eta)=-{ }^{C} D_{\alpha, \beta, \wp}^{\gamma} \frac{\partial T(\phi, \eta)}{\partial \phi}, \\
& \chi(\phi, \eta)=-{ }^{C} D_{\alpha, \beta, \wp}^{\gamma} \frac{\partial C(\phi, \eta)}{\partial \phi} .
\end{aligned}
$$

where ${ }^{C} D_{\alpha, \beta, \wp}^{\gamma}$ represents the Prabhakar fractional operator and detailed discussion with properties are given in [45]. Further, the classical Fourier's law will be obtained for $\beta=\gamma=0$. 


\subsection{Exact Solution of Temperature}

Applying Laplace transformation to Equations (9) and (18) to find the solution with conditions given in Equations (13)-(15), we have

$$
\operatorname{Pr} \bar{\zeta}(\phi, \xi)=-\frac{\partial \bar{q}(\phi, \xi)}{\partial \phi}
$$

and

$$
\bar{q}(\phi, \xi)=-\xi^{\beta}\left(1-\wp \xi^{-\alpha}\right)^{\gamma} \frac{\partial \bar{T}(\phi, \xi)}{\partial \phi}
$$

with

$$
\bar{T}(0, \xi)-\left.\omega \frac{\partial \bar{T}(\phi, \xi)}{\partial \phi}\right|_{\phi=0}=\bar{f}(\xi) \quad \text { and } \quad \bar{T}(\phi, \xi) \rightarrow 0 \text { as } \phi \rightarrow \infty
$$

where $\bar{\zeta}(\phi, \xi)$ represents the Laplace transformation of the function $\zeta(\phi, \eta)$ and defined as:

$$
\bar{\zeta}(\phi, \xi)=\int_{0}^{\infty} \zeta(\phi, \eta) e^{-\xi \eta} d \eta
$$

and $\xi$ is the transformed parameter.

Using Equation (21) into Equation (20), we find

$$
\begin{gathered}
\operatorname{Pr} \bar{\zeta}(\phi, \xi)=\xi^{\beta}\left(1-\wp \xi^{-\alpha}\right)^{\gamma} \frac{\partial^{2} \bar{T}(\phi, \xi)}{\partial \phi^{2}}, \\
\frac{\partial^{2} \bar{T}(\phi, \xi)}{\partial \phi^{2}}=\frac{\operatorname{Pr} \xi}{\xi^{\beta}\left(1-\wp \xi^{-\alpha}\right)^{\gamma}} \bar{T}(\phi, \xi), \\
\frac{\partial^{2} \bar{T}(\phi, \xi)}{\partial \phi^{2}}-A(\xi) \bar{T}(\phi, \xi)=0 .
\end{gathered}
$$

The solution for Equation (25) is written as:

$$
\bar{T}(\phi, \xi)=e_{1} e^{\phi \sqrt{A(\xi)}}+e_{2} e^{-\phi \sqrt{A(\xi)}}
$$

To determine the unknown constants $e_{1}$ and $e_{2}$, employing the stated conditions in Equation (22) for temperature, we have

$$
\bar{T}(\phi, \xi)=\frac{\bar{f}(\xi)}{1+\omega \sqrt{A(\xi)}} e^{-\phi \sqrt{A(\xi)}} .
$$

where $A(\xi)=\frac{\operatorname{Pr} \xi}{\xi^{\beta}\left(1-\wp \xi^{-\alpha}\right)^{\gamma}}$. We write the Equation (27) in series form by using the series formula for exponential function, then its equivalent form is expressed as:

$$
\begin{aligned}
\bar{T}(\phi, \xi) & =\bar{f}(\xi) \sum_{m=0}^{\infty}(-1)^{m}(\omega \sqrt{A(\xi)})^{m} \sum_{n=0}^{\infty} \frac{(-\phi \sqrt{A(\xi)})^{n}}{n !}, \\
& =\bar{f}(\xi) \sum_{m=0}^{\infty} \frac{(-\omega \sqrt{P r})^{m}}{\xi^{(\beta-1) \frac{m}{2}}\left(1-\wp \xi^{-\alpha}\right)^{\frac{\gamma m}{2}}} \sum_{n=0}^{\infty} \frac{(-\phi \sqrt{P r})^{n}}{n ! \xi^{(\beta-1) \frac{n}{2}}\left(1-\wp \xi^{-\alpha}\right)^{\frac{\gamma n}{2}}},
\end{aligned}
$$

Taking the inverse Laplace transformation of Equation (28), the required solution for temperature is written as: 


$$
T(\phi, \eta)=f(\eta) * \sum_{m=0}^{\infty}(-\omega)^{m}(\operatorname{Pr})^{\frac{m}{2}} \eta^{(\beta-1) \frac{m}{2}-1} E_{\alpha,(\beta-1) \frac{m}{2}}^{\frac{\gamma m}{2}}\left(\wp \eta^{\alpha}\right) * \sum_{n=0}^{\infty} \frac{(-\phi)^{n}}{n !}(\operatorname{Pr})^{\frac{n}{2}} \eta^{(\beta-1) \frac{n}{2}-1} E_{\alpha,(\beta-1) \frac{\eta n}{2}}^{\frac{\gamma n}{2}}\left(\wp \eta^{\alpha}\right) .
$$

where

$$
\mathscr{L}^{-1}\left\{\frac{1}{\xi^{\beta}\left(1-\wp \xi^{-\alpha}\right)^{\gamma}}\right\}=\mathscr{L}^{-1}\left\{\frac{\xi^{\alpha \gamma-\beta}}{\left(\xi^{\alpha}-\wp\right)^{\gamma}}\right\}=t^{\beta-1} E_{\alpha, \beta}^{\gamma}\left(\wp t^{\alpha}\right)
$$

and ' $*$ ' represents the convolution product.

\subsection{Exact Solution of Diffusion Equation}

Applying Laplace transformation to Equations (11) and (19) to find the solution with conditions given in Equations (13)-(15), we have

$$
\operatorname{Sc} \overline{\mathcal{C}}(\phi, \xi)=-\frac{\partial \bar{\chi}(\phi, \xi)}{\partial \phi}
$$

and

$$
\bar{\chi}(\phi, \xi)=-\xi^{\beta}\left(1-\wp \xi^{-\alpha}\right)^{\gamma} \frac{\partial \bar{C}(\phi, \xi)}{\partial \phi} .
$$

with

$$
\bar{C}(0, \xi)=\frac{1}{\xi} \quad \text { and } \quad \bar{C}(\phi, \xi) \rightarrow 0 \quad \text { as } \quad \phi \rightarrow \infty .
$$

Using Equation (31) into Equation (30), we find

$$
\begin{gathered}
\operatorname{Sc} \overline{\bar{C}}(\phi, \xi)=\xi^{\beta}\left(1-\wp \xi^{-\alpha}\right)^{\gamma} \frac{\partial^{2} \bar{C}(\phi, \xi)}{\partial \phi^{2}}, \\
\frac{\partial^{2} \bar{C}(\phi, \xi)}{\partial \phi^{2}}=\frac{S c \xi}{\xi^{\beta}\left(1-\wp \xi^{-\alpha}\right)^{\gamma}} \bar{C}(\phi, \xi), \\
\frac{\partial^{2} \bar{C}(\phi, \xi)}{\partial \phi^{2}}-B(\xi) \bar{C}(\phi, \xi)=0 .
\end{gathered}
$$

The solution for Equation (35) is written as:

$$
\bar{T}(\phi, \xi)=e_{3} e^{\phi \sqrt{B(\xi)}}+e_{4} e^{-\phi \sqrt{B(\xi)}} .
$$

To determine the unknown constants $e_{3}$ and $e_{4}$, employing the stated conditions in Equation (32) for concentration, we have

$$
\bar{C}(\phi, \xi)=\frac{1}{\xi} e^{-\phi \sqrt{B(\xi)}} .
$$

where $B(\xi)=\frac{S c \xi}{\xi^{\beta}\left(1-\wp \xi^{-\alpha}\right)^{\gamma}}$. We write the Equation (37) in series form by using the series formula for exponential function, then its equivalent form is expressed as: 


$$
\begin{aligned}
\bar{C}(\phi, \xi) & =\frac{1}{\xi} \sum_{k=0}^{\infty} \frac{(-\phi \sqrt{B(\xi)})^{k}}{k !}, \\
& =\frac{1}{\xi} \sum_{k=0}^{\infty} \frac{(-\phi \sqrt{S c})^{k}}{k ! \xi^{(\beta-1) \frac{k}{2}+1}\left(1-\wp \xi^{-\alpha}\right)^{\frac{\gamma k}{2}}} .
\end{aligned}
$$

Taking the inverse Laplace transformation of Equation (38), the required solution for concentration is written as:

$$
C(\phi, \eta)=\sum_{k=0}^{\infty} \frac{(-\phi)^{k}}{k !}(S c)^{\frac{k}{2}} \eta^{(\beta-1) \frac{k}{2}} E_{\alpha,(\beta-1) \frac{\gamma k}{2}+1}^{\frac{k}{2}}\left(\wp \eta^{\alpha}\right) .
$$

\subsection{Exact Solution of Fluid Velocity}

The velocity field solution from Equation (8) with the help of Laplace transformation is calculated as:

$$
(1+\lambda \xi) \xi \bar{u}(\phi, \xi)=\frac{d^{2} \bar{u}(\phi, \xi)}{d \phi^{2}}+(1+\lambda \xi) G r \bar{T}(\phi, \xi)+(1+\lambda \xi) G m \bar{C}(\phi, \xi)
$$

with

$$
\bar{u}(0, \xi)=0 \text { and } \bar{u}(\phi, \xi) \rightarrow 0 \quad \text { as } \quad \phi \rightarrow \infty .
$$

substituting the value of $\bar{T}(\phi, \xi)$ from Equation (27) and the value of $\bar{C}(\phi, \xi)$ from Equation (37) in Equation (40), then after manipulation the solution written in the form

$$
\begin{aligned}
\bar{u}(\phi, \xi) & =e_{5} e^{\phi \sqrt{\xi(1+\lambda \xi)}}+e_{6} e^{-\phi \sqrt{\xi(1+\lambda \xi)}}-(1+\lambda \xi) G r\left[\frac{\bar{f}(\xi)}{1+\omega \sqrt{A(\xi)}}\right]\left[\frac{e^{-\phi \sqrt{A(\xi)}}}{A(\xi)-\xi(1+\lambda \xi)}\right] \\
& -(1+\lambda \xi) G m \frac{1}{\xi}\left[\frac{e^{-\phi \sqrt{B(\xi)}}}{B(\xi)-\xi(1+\lambda \xi)}\right] .
\end{aligned}
$$

The involving constants $e_{5}$ and $e_{6}$ in the above Equation (42) are determined with the help of stated conditions in Equation (41), then solution is written as:

$$
\begin{aligned}
\bar{u}(\phi, \xi) & =(1+\lambda \xi) G r\left[\frac{\bar{f}(\xi)}{1+\omega \sqrt{A(\xi)}}\right]\left[\frac{e^{-\phi \sqrt{A(\xi)}}-e^{-\phi \sqrt{\xi(1+\lambda \xi)}}}{\xi(1+\lambda \xi)-A(\xi)}\right] \\
& +(1+\lambda \xi) G m \frac{1}{\xi}\left[\frac{e^{-\phi \sqrt{B(\xi)}}-e^{-\phi \sqrt{\xi(1+\lambda \xi)}}}{\xi(1+\lambda \xi)-B(\xi)}\right], \\
& =\frac{G r(1+\lambda \xi)}{\xi(1+\lambda \xi)-A(\xi)}\left[\frac{\bar{f}(\xi) e^{-\phi \sqrt{A(\xi)}}}{1+\omega \sqrt{A(\xi)}}-\frac{\bar{f}(\xi) e^{-\phi \sqrt{\xi(1+\lambda \xi)}}}{1+\omega \sqrt{A(\xi)}}\right] \\
& +\frac{G m(1+\lambda \xi)}{\xi(1+\lambda \xi)-B(\xi)}\left[\frac{e^{-\phi \sqrt{B(\xi)}}}{\xi}-\frac{e^{-\phi \sqrt{\xi(1+\lambda \xi)}}}{\xi}\right] .
\end{aligned}
$$

Equation (43) can also be written in a more precise form as:

$$
\bar{u}(\phi, \xi)=G r \bar{u}_{1}(\phi, \xi)\left[\bar{T}(\phi, \xi)-\bar{f}(\xi) \bar{u}_{2}(\phi, \xi)\right]+G m \bar{u}_{3}(\phi, \xi)\left[\bar{C}(\phi, \xi)-\bar{u}_{4}(\phi, \xi)\right] .
$$

Taking the Laplace inverse transformation along with the convolution theorem, the velocity field solution is finally obtained as: 


$$
\begin{aligned}
& u(\phi, \eta)=G r u_{1}(\phi, \eta) *\left[T(\phi, \eta)-f(\eta) * u_{2}(\phi, \eta)\right]+G m u_{3}(\phi, \eta) *\left[C(\phi, \eta)-u_{4}(\phi, \eta)\right] . \\
& \text { where } \\
& u_{1}(\phi, \eta)=\mathscr{L}^{-1}\left\{\bar{u}_{1}(\phi, \xi)\right\}=\mathscr{L}^{-1}\left\{\frac{(1+\lambda \xi)}{\xi(1+\lambda \xi)-A(\xi)}\right\} \\
& =\mathscr{L}^{-1}\left\{\sum_{k=0}^{\infty} \sum_{r=0}^{\infty} \frac{(P r)^{k}(-\lambda)^{r} \Gamma(k+r)}{r ! \Gamma(k)} \frac{1}{\xi^{(\beta k-r+1)}\left(1-\wp \xi^{-\alpha}\right)^{\gamma k}}\right\} \\
& =\sum_{k=0}^{\infty} \sum_{r=0}^{\infty} \frac{(P r)^{k}(-\lambda)^{r} \Gamma(k+r)}{r ! \Gamma(k)} \eta^{\beta k-r} E_{\alpha, \beta k-r+1}^{\gamma k}\left(\wp \eta^{\alpha}\right) \\
& u_{2}(\phi, \eta)=\mathscr{L}^{-1}\left\{\bar{u}_{2}(\phi, \xi)\right\}=\mathscr{L}^{-1}\left\{\frac{e^{-\phi \sqrt{\xi(1+\lambda \xi)}}}{1+\omega \sqrt{A(\xi)}}\right\} \\
& =\mathscr{L}^{-1}\left\{\left(\sum_{m=0}^{\infty} \frac{(-\omega \sqrt{\operatorname{Pr}})^{m}}{\xi^{(\beta-1) \frac{m}{2}}\left(1-\wp \xi^{-\alpha}\right)^{\frac{\gamma m}{2}}}\right)\left(\sum_{n=0}^{\infty} \sum_{i=0}^{\infty} \frac{(-\phi)^{n}(\lambda)^{\frac{n}{2}-i} \Gamma\left(\frac{n}{2}+1\right)}{n ! i ! \Gamma\left(\frac{n}{2}-i+1\right)} \frac{1}{\xi^{i-n}}\right)\right\} \\
& =\left(\sum_{m=0}^{\infty}(-\omega)^{m}(P r)^{\frac{m}{2}} \eta^{(\beta-1) \frac{m}{2}-1} E_{\alpha,(\beta-1) \frac{m}{2}}^{\frac{\gamma m}{2}}\left(\wp \eta^{\alpha}\right)\right) *\left(\sum_{n=0}^{\infty} \sum_{i=0}^{\infty} \frac{(-\phi)^{n}(\lambda)^{\frac{n}{2}-i} \Gamma\left(\frac{n}{2}+1\right)}{n ! i ! \Gamma\left(\frac{n}{2}-i+1\right)} \frac{\eta^{i-n-1}}{\Gamma(i-n)}\right), \\
& u_{3}(\phi, \eta)=\mathscr{L}^{-1}\left\{\bar{u}_{3}(\phi, \xi)\right\}=\mathscr{L}^{-1}\left\{\frac{(1+\lambda \xi)}{\xi(1+\lambda \xi)-B(\xi)}\right\} \\
& =\mathscr{L}^{-1}\left\{\sum_{k=0}^{\infty} \sum_{r=0}^{\infty} \frac{(S c)^{k}(-\lambda)^{r} \Gamma(k+r)}{r ! \Gamma(k)} \frac{1}{\xi^{(\beta k-r+1)}\left(1-\wp \xi^{-\alpha}\right)^{\gamma k}}\right\} \\
& =\sum_{k=0}^{\infty} \sum_{r=0}^{\infty} \frac{(S c)^{k}(-\lambda)^{r} \Gamma(k+r)}{r ! \Gamma(k)} \eta^{\beta k-r} E_{\alpha, \beta k-r+1}^{\gamma k}\left(\wp \eta^{\alpha}\right), \\
& u_{4}(\phi, \eta)=\mathscr{L}^{-1}\left\{\bar{u}_{4}(\phi, \xi)\right\}=\mathscr{L}^{-1}\left\{\frac{1}{\xi} e^{-\phi \sqrt{\xi(1+\lambda \xi)}}\right\} \\
& =\mathscr{L}^{-1}\left\{\sum_{n=0}^{\infty} \sum_{\delta=0}^{\infty} \frac{(-\phi)^{n}(\lambda)^{\frac{n}{2}-\delta} \Gamma\left(\frac{n}{2}+1\right)}{n ! \delta ! \Gamma\left(\frac{n}{2}-\delta+1\right)} \frac{1}{\xi^{\delta-n+1}}\right\} \\
& =\sum_{n=0}^{\infty} \sum_{\delta=0}^{\infty} \frac{(-\phi)^{n}(\lambda)^{\frac{n}{2}-\delta} \Gamma\left(\frac{n}{2}+1\right)}{n ! \delta ! \Gamma\left(\frac{n}{2}-\delta+1\right)} \frac{\eta^{\delta-n}}{\Gamma(\delta-n+1)}
\end{aligned}
$$

\subsubsection{Classical Maxwell Fluid}

To find the Ordinary Maxwell fluid, we substitute $\beta=0$ and $\gamma=0$ in Equation (43), then the transformed velocity expression becomes

$$
\begin{aligned}
\bar{u}(\phi, \xi) & =\frac{G r(1+\lambda \xi)}{\xi(1+\lambda \xi)-\operatorname{Pr} \xi}\left[\frac{\bar{f}(\xi) e^{-\phi \sqrt{\operatorname{Pr} \xi}}}{1+\omega \sqrt{\operatorname{Pr} \xi}}-\frac{\bar{f}(\xi) e^{-\phi \sqrt{\xi(1+\lambda \xi)}}}{1+\omega \sqrt{\operatorname{Pr} \xi}}\right] \\
& +\frac{G m(1+\lambda \xi)}{\xi(1+\lambda \xi)-S c \xi}\left[\frac{e^{-\phi \sqrt{S c \xi}}}{\xi}-\frac{e^{-\phi \sqrt{\xi(1+\lambda \xi)}}}{\xi}\right]
\end{aligned}
$$

\subsubsection{Fractionalized Viscous Fluid}

For this case, we take $\lambda=0$ in Equation (43); then the velocity expression for viscous fluid is written as 
$\bar{u}(\phi, \xi)=\frac{G r}{\xi-A(\xi)}\left[\frac{\bar{f}(\xi) e^{-\phi \sqrt{A(\xi)}}}{1+\omega \sqrt{A(\xi)}}-\frac{\bar{f}(\xi) e^{-\phi \sqrt{\xi}}}{1+\omega \sqrt{A(\xi)}}\right]+\frac{G m}{\xi-B(\xi)}\left[\frac{e^{-\phi \sqrt{B(\xi)}}}{\xi}-\frac{e^{-\phi \sqrt{\xi}}}{\xi}\right]$

\subsubsection{Ordinary Viscous Fluid}

For this case, we take $\lambda=0$ in Equation (46); then the velocity expression for classical viscous fluid is written as

$$
\bar{u}(\phi, \xi)=\frac{G r}{\xi-\operatorname{Pr} \xi}\left[\frac{\bar{f}(\xi) e^{-\phi \sqrt{\operatorname{Pr} \xi}}}{1+\omega \sqrt{\operatorname{Pr} \xi}}-\frac{\bar{f}(\xi) e^{-\phi \sqrt{\xi}}}{1+\omega \sqrt{\operatorname{Pr} \xi}}\right]+\frac{G m}{\xi-S c \xi}\left[\frac{e^{-\phi \sqrt{S c \xi}}}{\xi}-\frac{e^{-\phi \sqrt{\xi}}}{\xi}\right]
$$

We recover the same velocity field expressions for all cases which are discussed above by taking $G m=0$ in Equations (43), (46)-(48) as X. H. Zhang et al. [44] investigated in Equations (26), (33), (35) and (37). All these results validate our current results.

\section{Results and Discussion}

In the present work, we investigated the time dependent, in-compressible, electrically conducting natural convective movement of Maxwell fluid over an erected plate with infinite length along with a wall slip condition on temperature under constant concentration. For the sake of generalized memory effects, a fractional model was developed by applying the newly introduced Prabhakar fractional operator and having a Mittag-Leffler kernel in the constitutive equations. This fractional model has been solved analytically and exact solutions for dimensionless velocity, concentration, and energy equations were calculated in terms of Mittag-Leffler functions by employing the Laplace transformation. The influence of the various system parameters such as $\alpha, \operatorname{Pr}, \beta, S c, G r, \gamma$, and $G m$ are used to discuss the physical interpretation of the derived results. The analytical solutions for energy, concentration, and momentum equations are graphically portrayed in Figures 2-18.
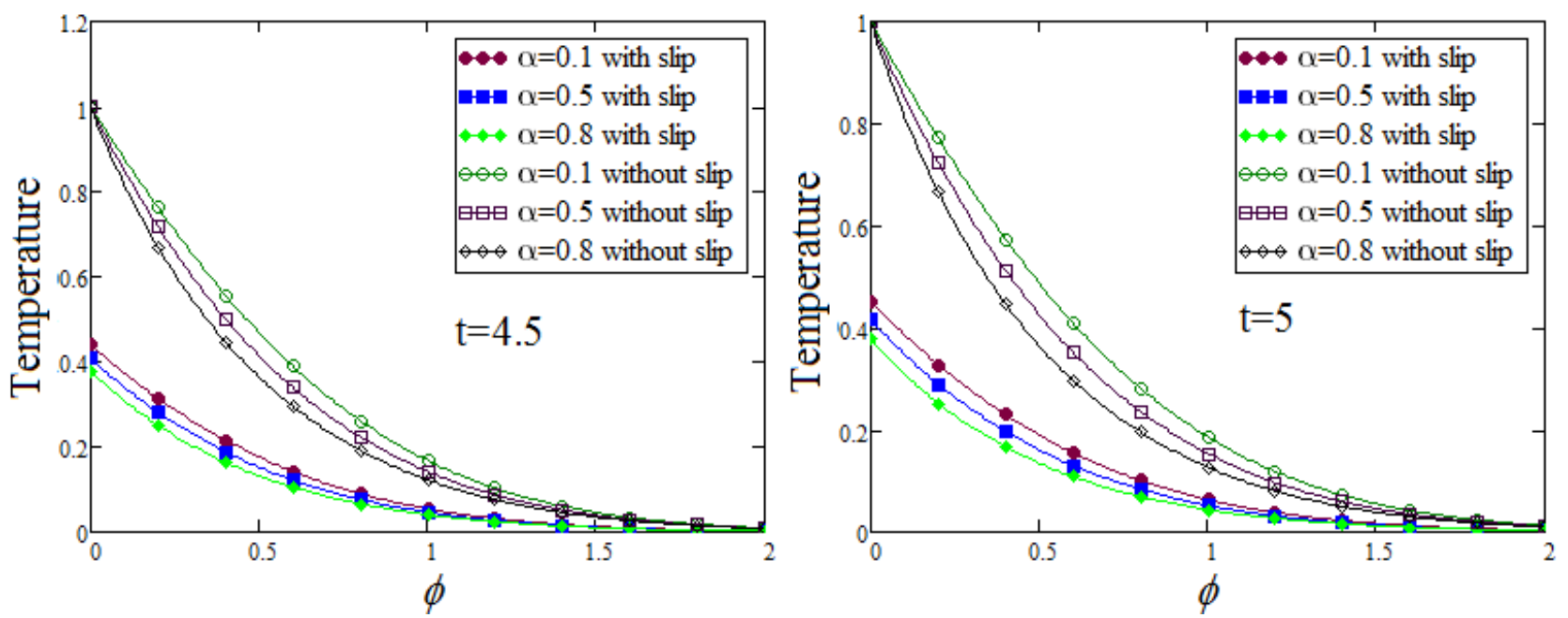

Figure 2. Graphical representations of temperature profile in the presence of slip and no slip conditions by taking the distinct values of $\alpha$ at two different levels of time, when $\operatorname{Pr}=12, \beta=0.3, \wp=0.4$, $\omega=0.5$, and $\gamma=0.5$.

Figures 2-4 portray the effect of fractional parameters $\alpha, \beta$, and $\gamma$ on temperature profile by taking two distinct values of time in the presence of slip condition and taking the value of slip parameter to be zero. From these graphs, it is observed that the decline in temperature profile corresponds to large values of fractional parameters. Further, there is a notable, rapid decline in temperature on the profile with the slip condition compared to without slip condition. It is also analyzed that fractional parameters have a significant effect 
on thermal flux for smaller values of time, but the effect is more significant on thermal flux for large values of time.
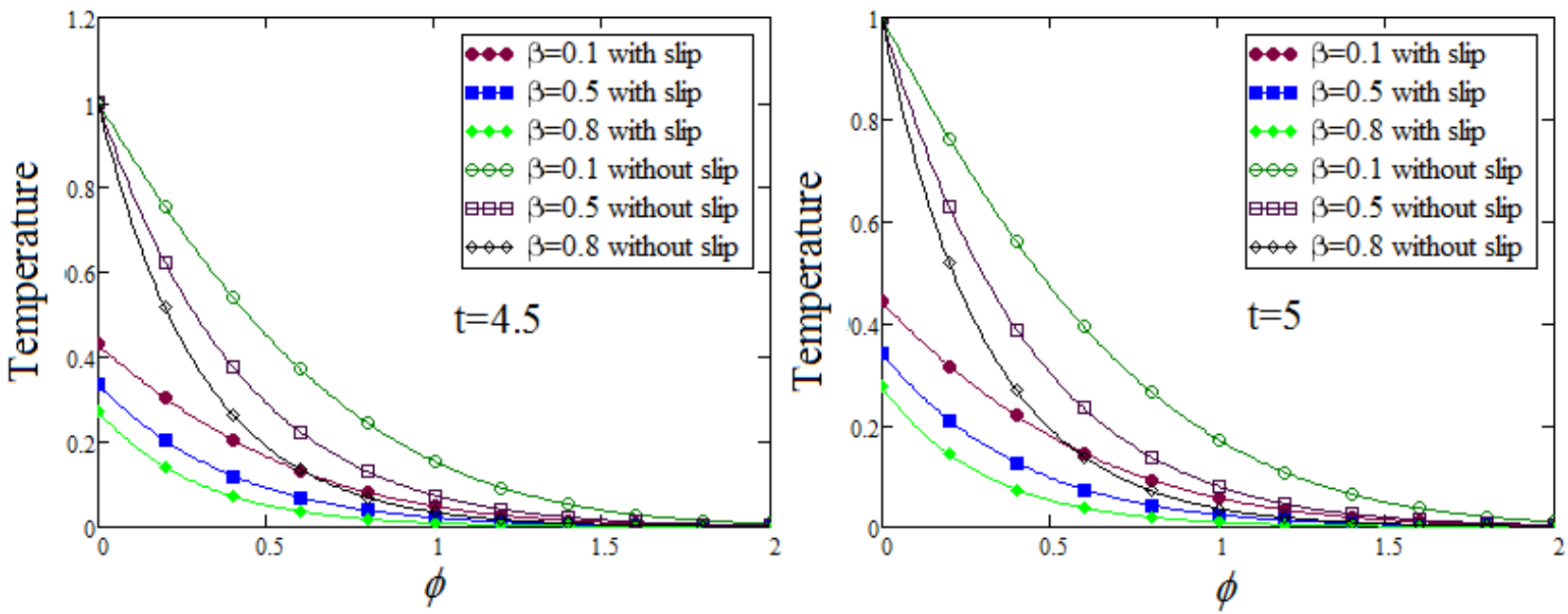

Figure 3. Graphical representations of temperature profile in the presence of slip and no slip conditions by taking the distinct values of $\beta$ at two different levels of time, when $\operatorname{Pr}=12, \omega=0.5, \wp=0.4$, $\alpha=0.3$, and $\gamma=0.5$.
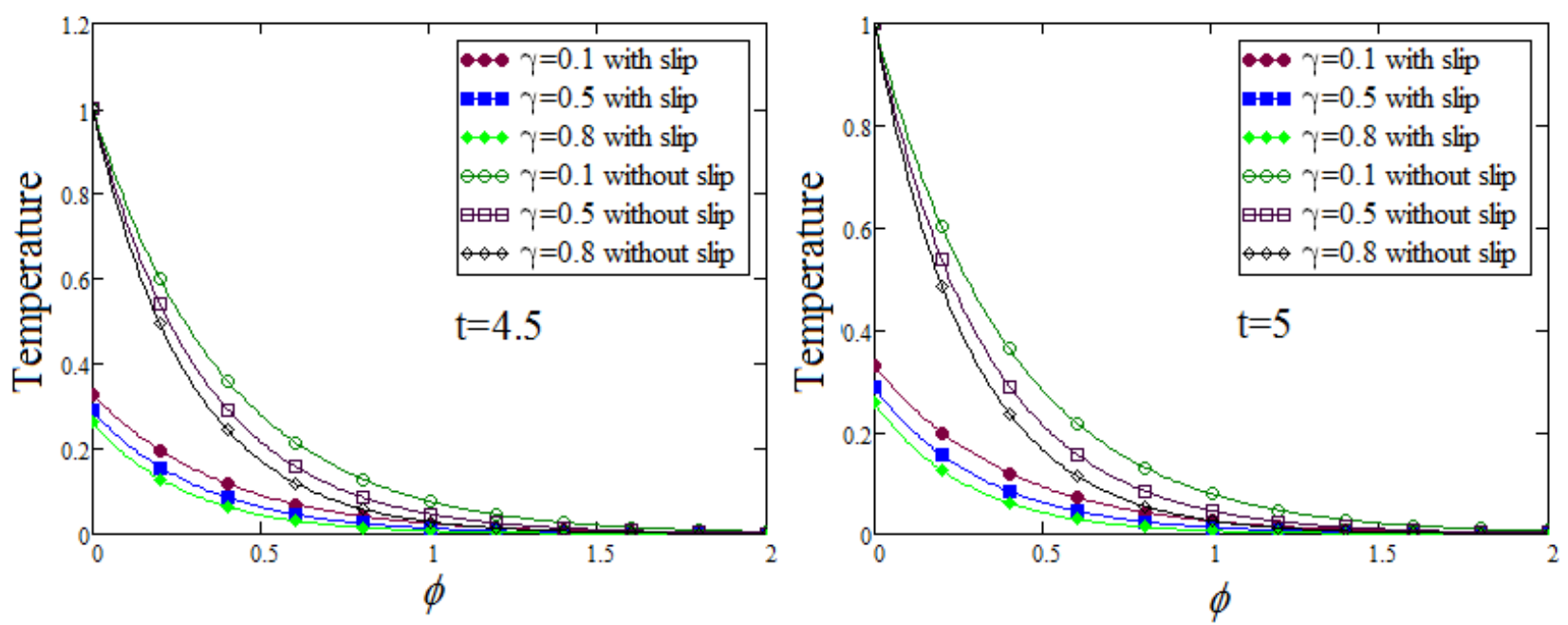

Figure 4. Graphical representations of temperature profile in the presence of slip and no slip conditions by taking the distinct values of $\gamma$ at two different levels of time, when $\operatorname{Pr}=12, \omega=0.5, \wp=0.4$, $\beta=0.3$, and $\alpha=0.5$.

Figure 5 displayed the impact of Prandtl number $P_{r}$ over the temperature profile by taking the various values of $\mathrm{Pr}$ at two different levels of time. A decay in temperature profile is seen while increasing the values of Prandtl number with and without slip conditions. Physically, when the values of $P r$ increase, then the thermal boundary layer thickness decreases rapidly, which causes a decrease in energy profile.

Figures 6-8 illustrated the behavior of $\alpha, \beta$, and $\gamma$ respectively, on mass profile by taking two distinct values of time. From these curves, it is noted that a decay in concentration profile corresponds to large values of fractional parameters. It is also seen that fractional parameters have a significant effect on mass profile for smaller values of time, but the effect is more significant for large values of time. 

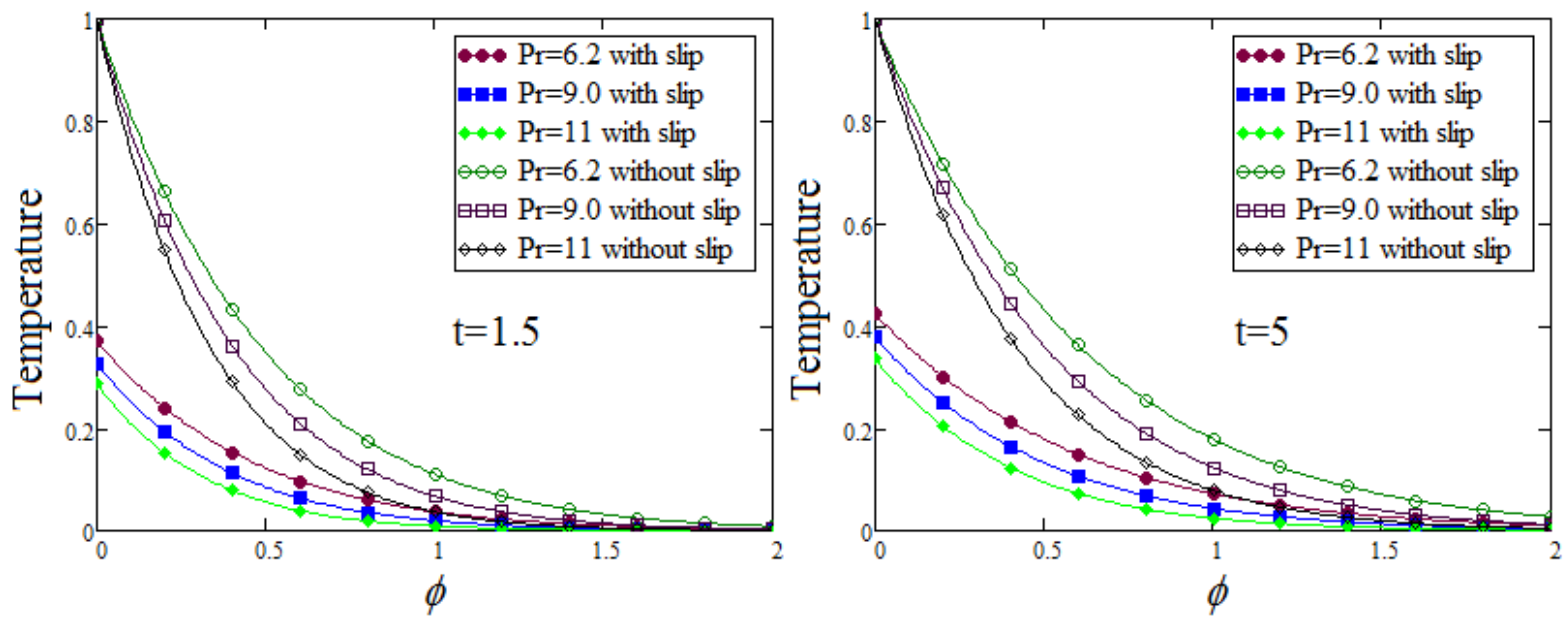

Figure 5. Graphical representations of temperature profile in the presence of slip and no slip conditions by taking the distinct values of $\operatorname{Pr}$ at two different levels of time, when $\alpha=0.4, \beta=0.3, \wp=0.4$, $\omega=0.5$, and $\gamma=0.5$.
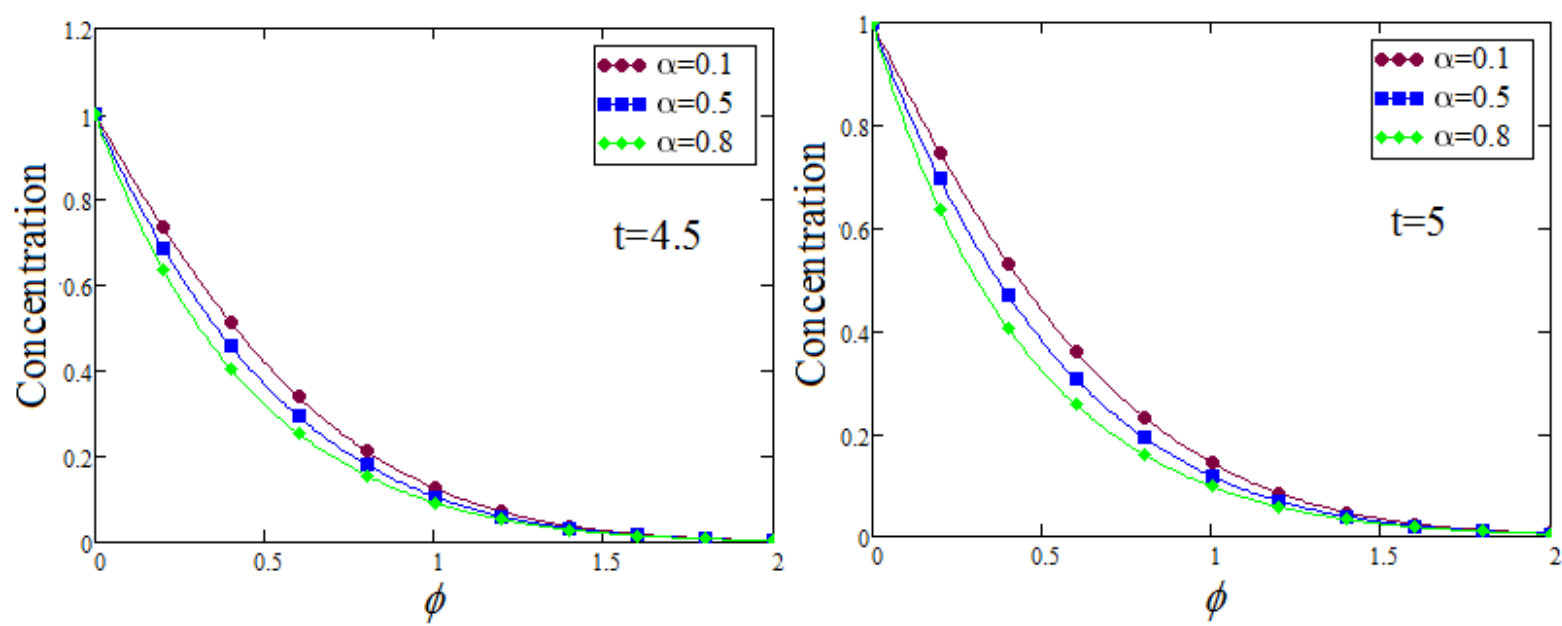

Figure 6. Graphical representations of concentration profile by taking distinct values of $\alpha$ at two different levels of time, when $S c=9, \wp=0.4, \beta=0.3$ and $\gamma=0.5$.
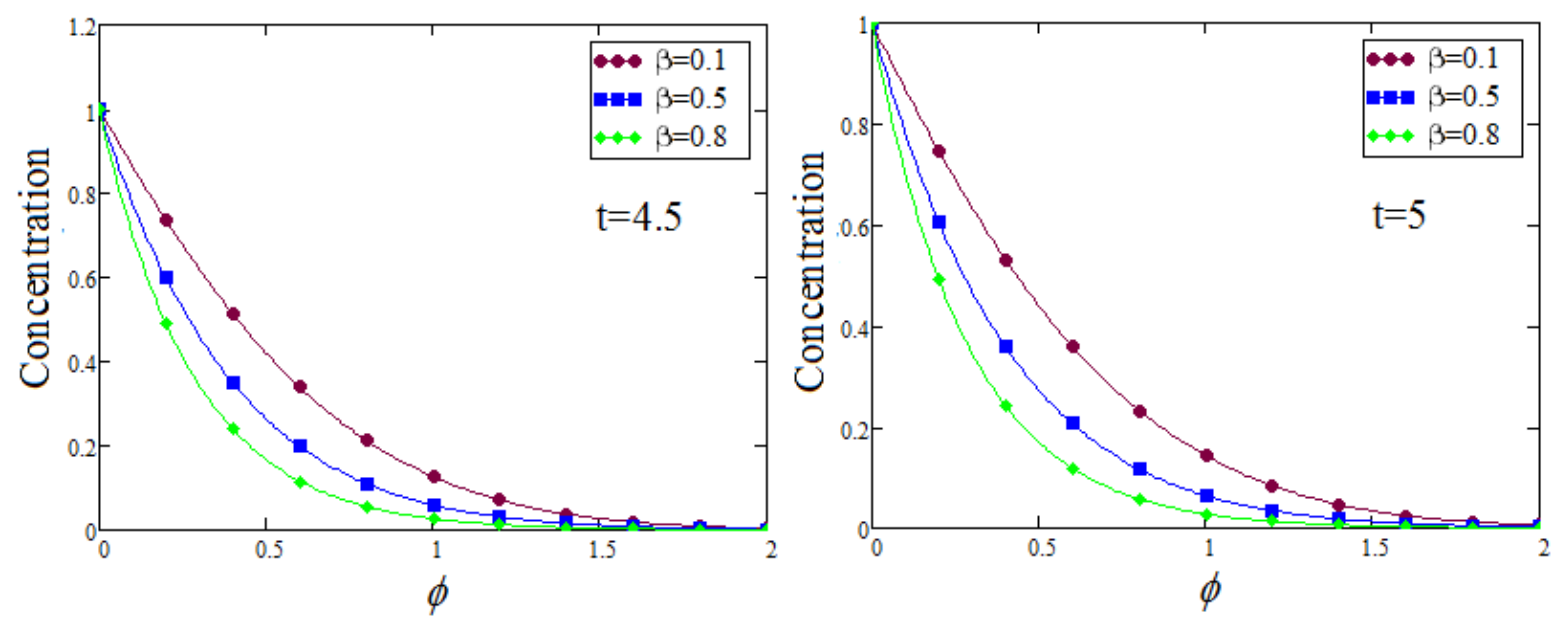

Figure 7. Graphical representations of concentration profile by taking distinct values of $\beta$ at two different levels of time, when $S c=9, \wp=0.4, \alpha=0.3$, and $\gamma=0.5$. 

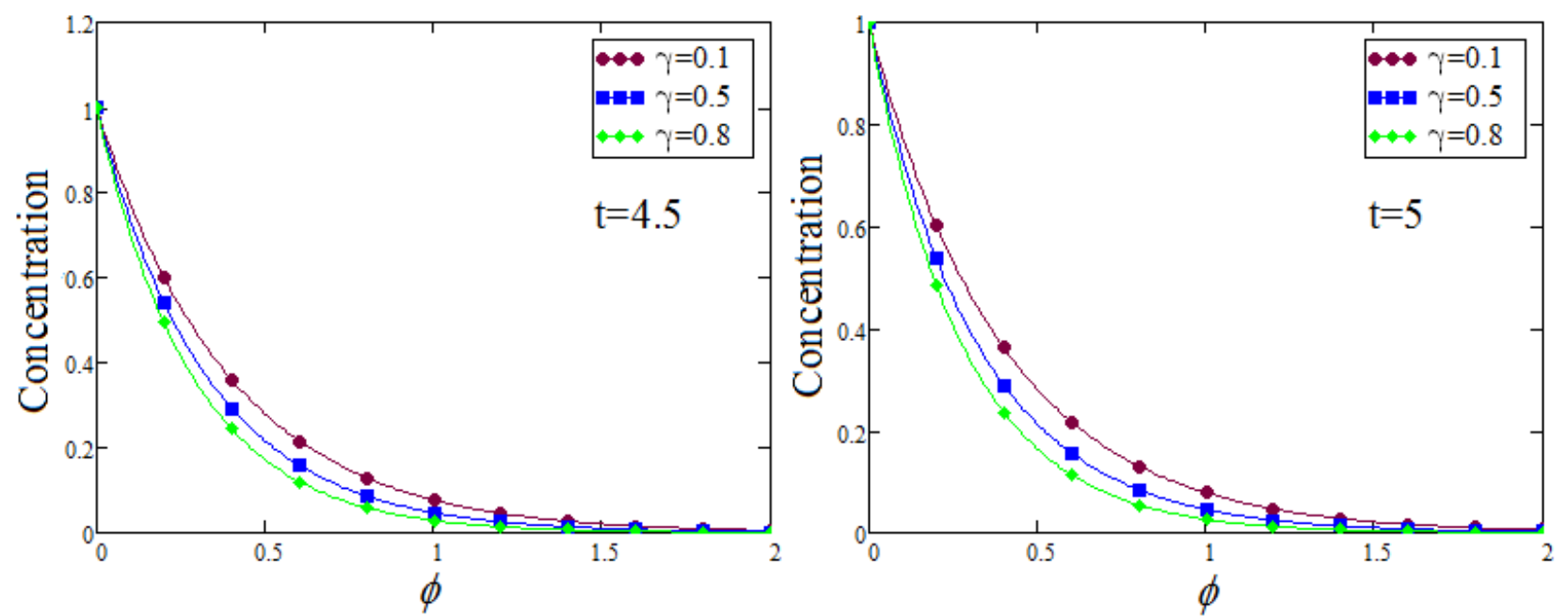

Figure 8. Graphical representations of concentration profile by taking distinct values of $\gamma$ at two different levels of time, when $S c=9, \wp=0.4, \beta=0.3$, and $\alpha=0.5$.

Figure 9 displayed the influence of Schmidt number $S c$ over the concentration profile by taking the various values of $S c$ corresponding to small and large values of time. It is noted that a decay in mass profile occurs while increasing the values of Schmidt number.

Figures 10-12 were plotted to analyze the behavior of $\alpha, \beta$, and $\gamma$ on a velocity contour along with the slip and no slip conditions against two dissimilar values of time. It is depicted from these graphs that velocity profile decreases due to enhanced values of fractional parameters. Further, it is notable that the graph of fluid velocity without slip condition is higher as compared to the graph of fluid velocity with slip conditions.

Figure 13 displayed the impact of Prandtl number Pr over the velocity field by taking the various values of $P r$ at two different levels of time. It is noted that decay in velocity profile occurs while increasing the values of Prandtl number for both slip and no slip conditions. Physically, when the values of $P r$ increase, then the thermal boundary layer thickness decreases rapidly which causes a decrease in the momentum profile.
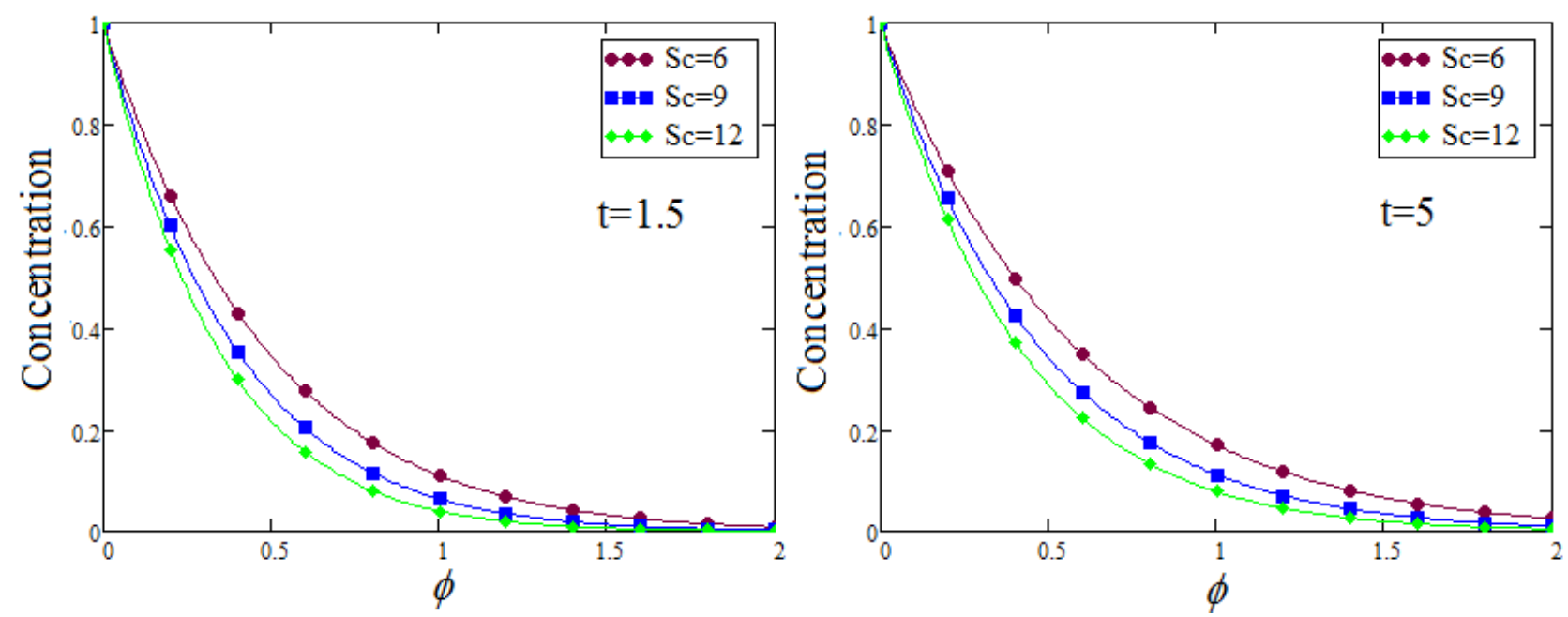

Figure 9. Graphical representations of concentration profile by taking distinct values of $S c$ at two different levels of time, when $\alpha=0.5, \wp=0.4, \beta=0.3$, and $\gamma=0.5$. 

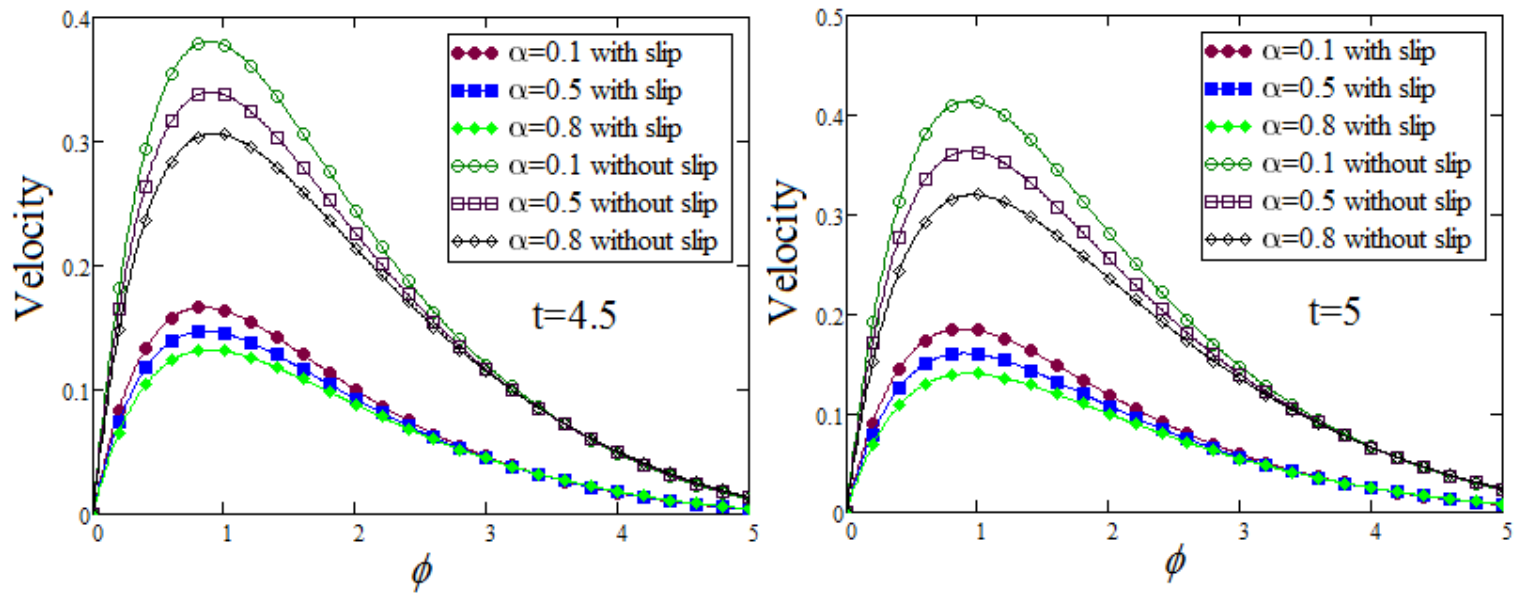

Figure 10. Graphical representations of velocity profile in the presence of slip and no slip conditions by taking the dissimilar values of $\alpha$ at two different levels of time, when $G r=5, G m=3.5, \wp=0.4$, $\operatorname{Pr}=12, \omega=0.5, S c=9, \gamma=0.3$, and $\beta=0.5$.
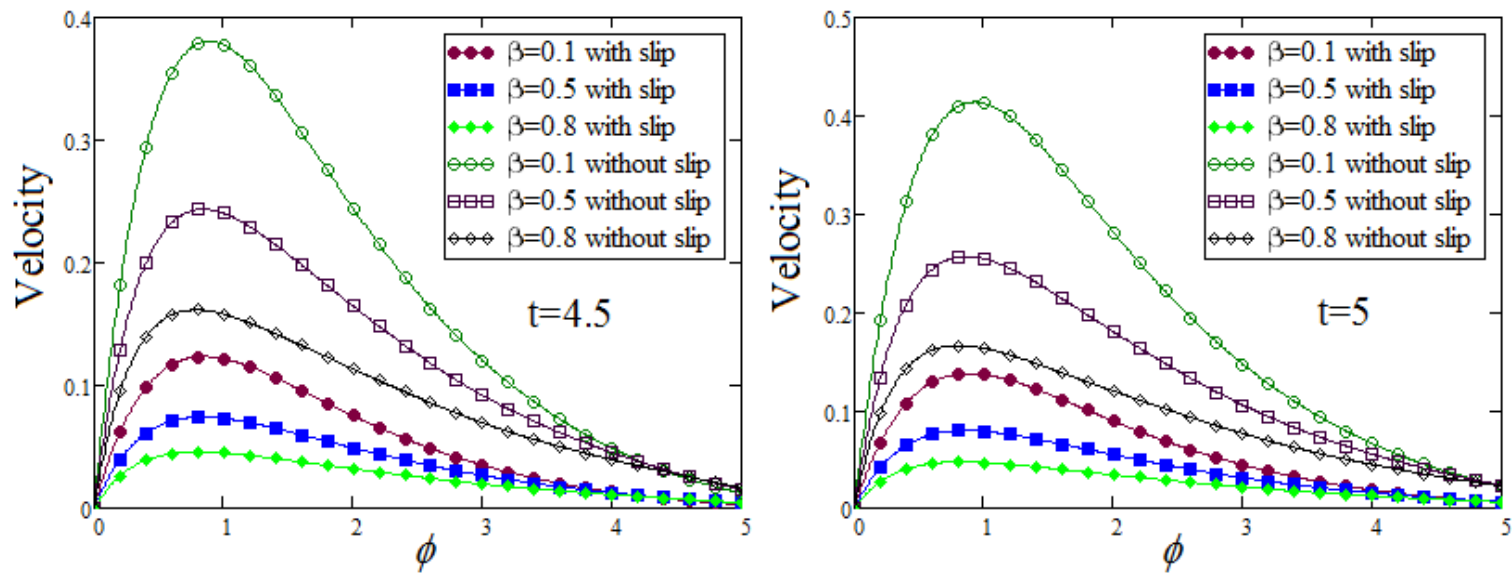

Figure 11. Graphical representations of velocity profile in the presence of slip and no slip conditions by taking the dissimilar values of $\beta$ at two different levels of time, when $G r=5, G m=3.5, \wp=0.4$, $\operatorname{Pr}=12, \omega=0.5, S_{c}=9, \gamma=0.3$, and $\alpha=0.5$.
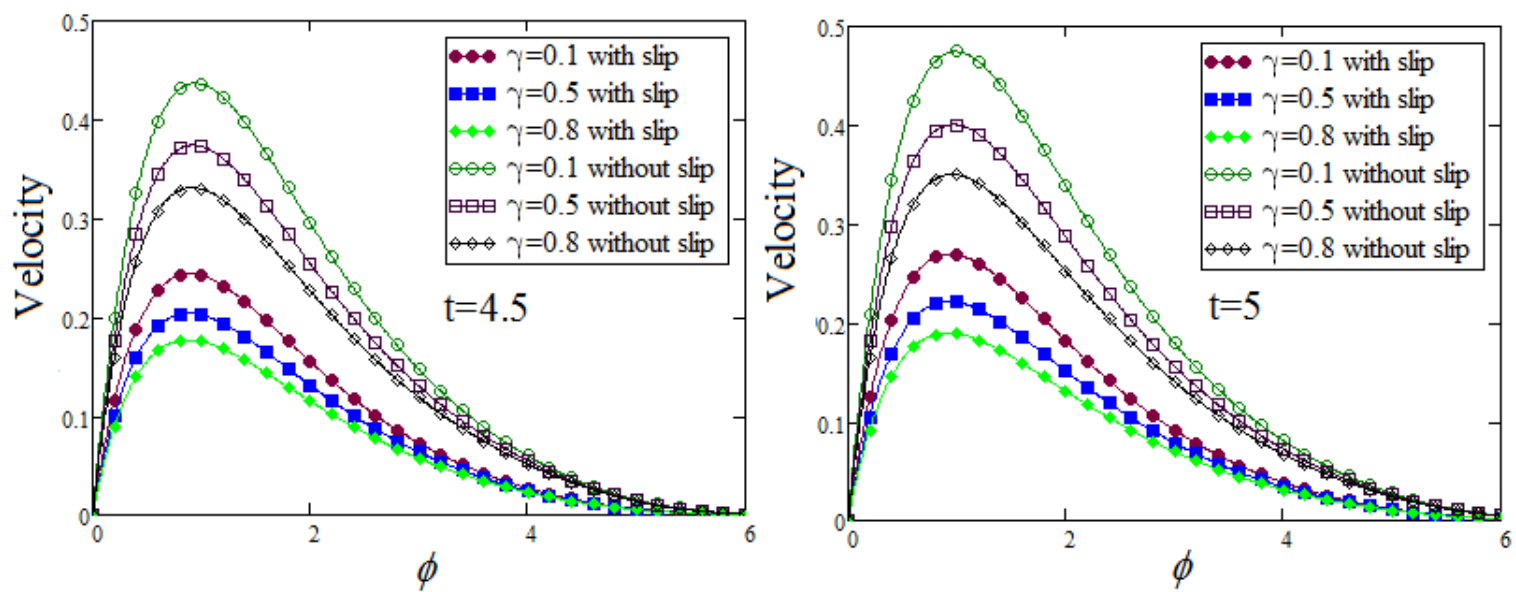

Figure 12. Graphical representations of velocity profile in the presence of slip and no slip conditions by taking the dissimilar values of $\gamma$ at two different levels of time, when $G r=5, G m=3.5, \wp=0.4$, $\operatorname{Pr}=12, \omega=0.5, S c=9, \beta=0.3$, and $\alpha=0.5$. 
Figures 14 and 15 exemplify the velocity graphs to interpret the impact of thermal and mass Grashof numbers $G r$ and $G m$, respectively. An increase in the velocity curve appeared due to a boost in the values of $\mathrm{Gr}$ and $\mathrm{Gm}$.

Figure 16 represented the influence of Schmidt number $S c$ over the velocity profile by taking the various values of $S c$ corresponding to small and large values of time by considering the cases with slip and no slip conditions. We detected a decline in the velocity profile while increasing the values of the Schmidt number for both slip and no slip conditions.
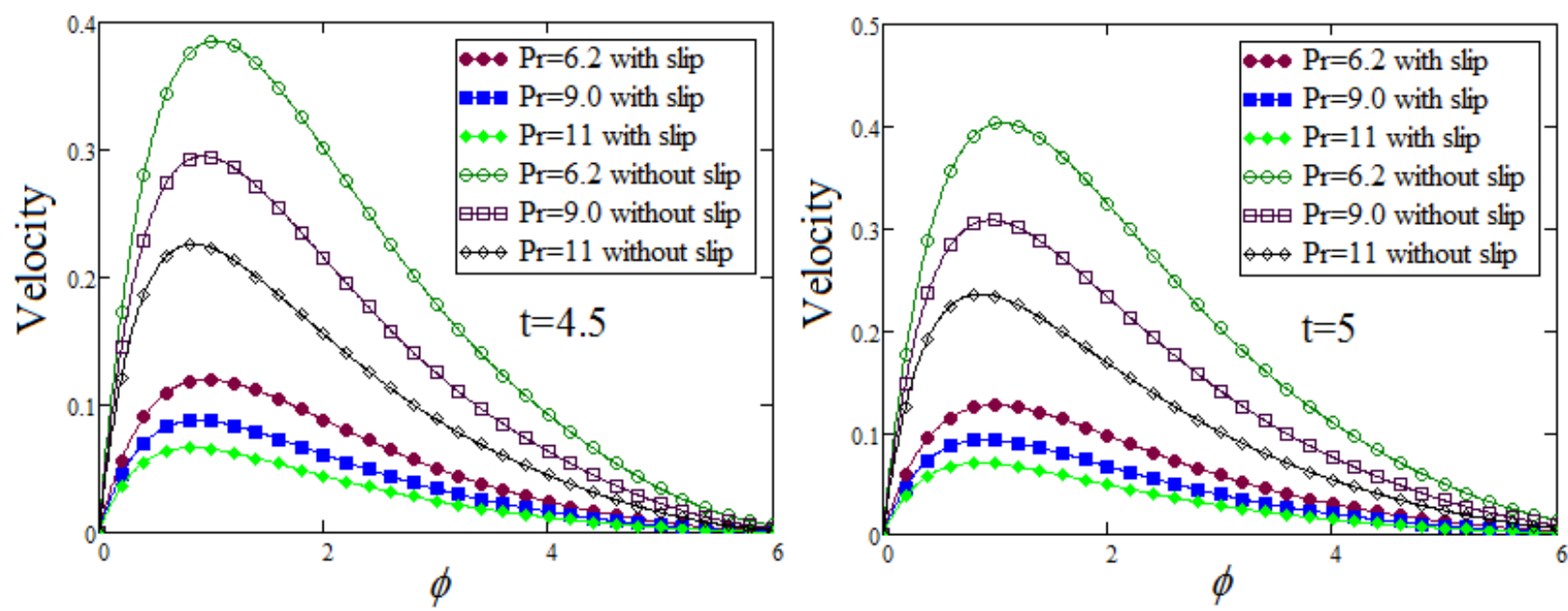

Figure 13. Graphical representations of velocity profile in the presence of slip and no slip conditions by taking the dissimilar values of $P r$ at two different levels of time, when $G r=5, G m=3.5, \wp=0.4$, $\omega=0.5, S c=9, \alpha=0.5 \beta=0.3$, and $\gamma=0.4$.
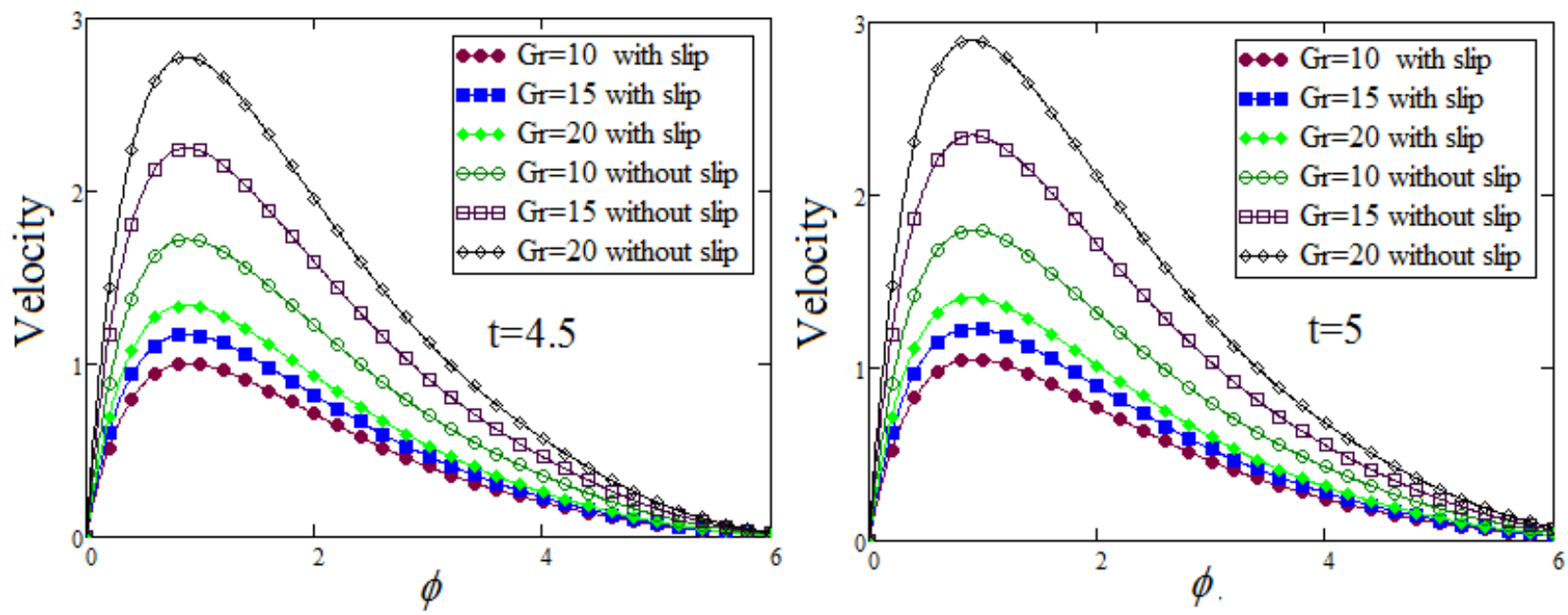

Figure 14. Graphical representations of velocity profile in the presence of slip and no slip conditions by taking the dissimilar values of $G r$ at two different levels of time, when $\operatorname{Pr}=12, G m=3.5, \wp=0.4$, $\omega=0.5, S c=9, \alpha=0.5 \beta=0.3$, and $\gamma=0.4$. 

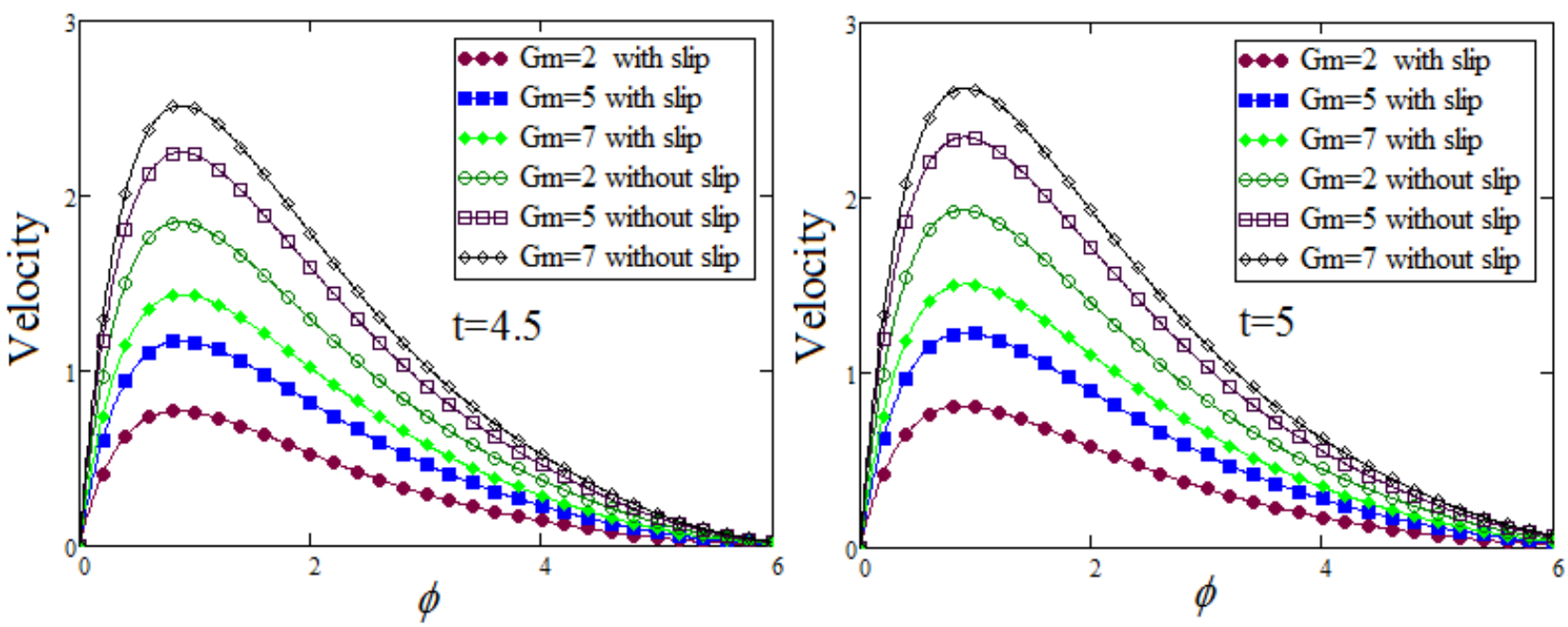

Figure 15. Graphical representations of velocity profile in the presence of slip and no slip conditions by taking the dissimilar values of $G m$ at two different levels of time, when $G r=5, \operatorname{Pr}=11, \wp=0.4$, $\omega=0.5, S c=9, \alpha=0.5 \beta=0.3$, and $\gamma=0.4$.
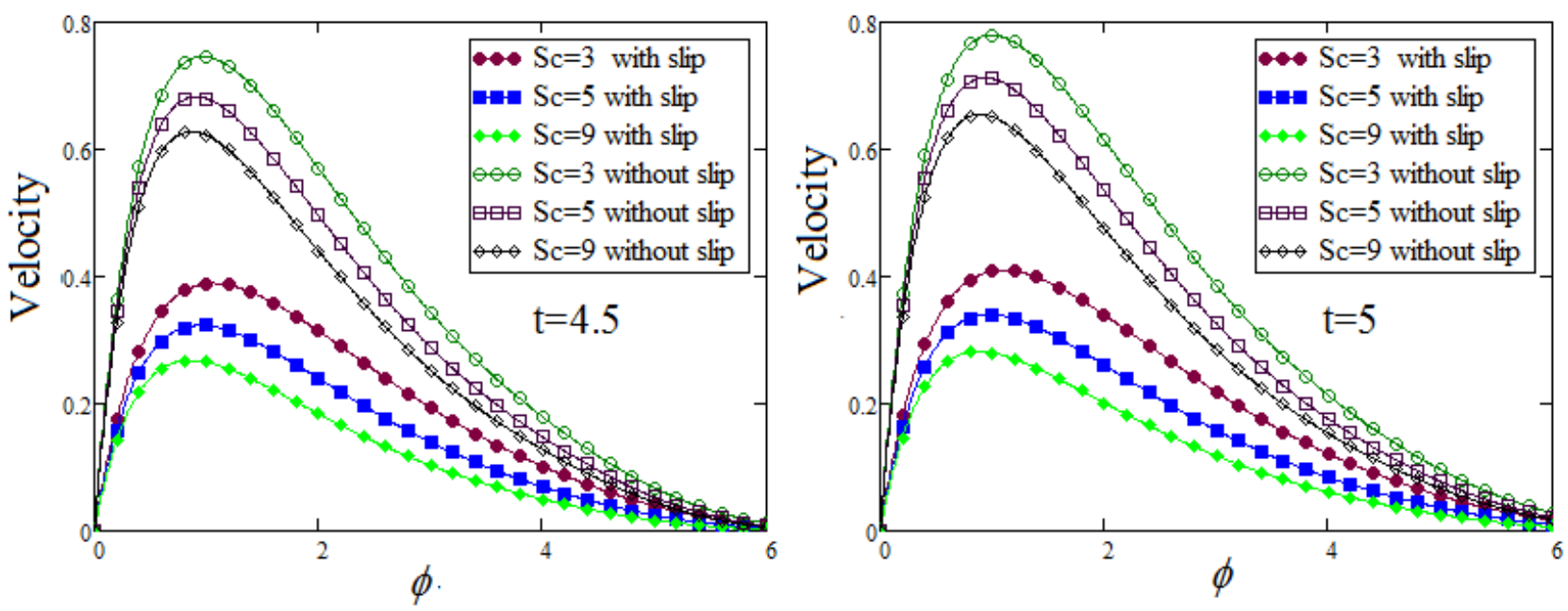

Figure 16. Graphical representations of velocity profile in the presence of slip and no slip conditions by taking the dissimilar values of $S c$ at two different levels of time, when $G r=5, G m=3.5, \wp=0.4$, $\omega=0.5, \operatorname{Pr}=9, \alpha=0.5 \beta=0.3$, and $\gamma=0.4$.

Figures 17 and 18 were plotted to compare different fluids such as the fractional Maxwell, classical Maxwell, fractional viscous, and classical viscous fluid models, along with and without slip conditions at the boundary for two distinct levels of time. It is eminent to point out that the movement of the Maxwell fluids for both fractional and classical cases are faster as compared to viscous fluids for ordinary as well as fractional cases. Furthermore, from these graphs, it is visualized that ordinary Maxwell fluid and ordinary viscous fluid have relatively higher velocity as compared to fractional Maxwell fluid and fractional viscous fluid. Additionally, it is important to mention that, for classical and fractional models, the velocity field perceives identical behavior for the cases of both slip and zero slip conditions. 

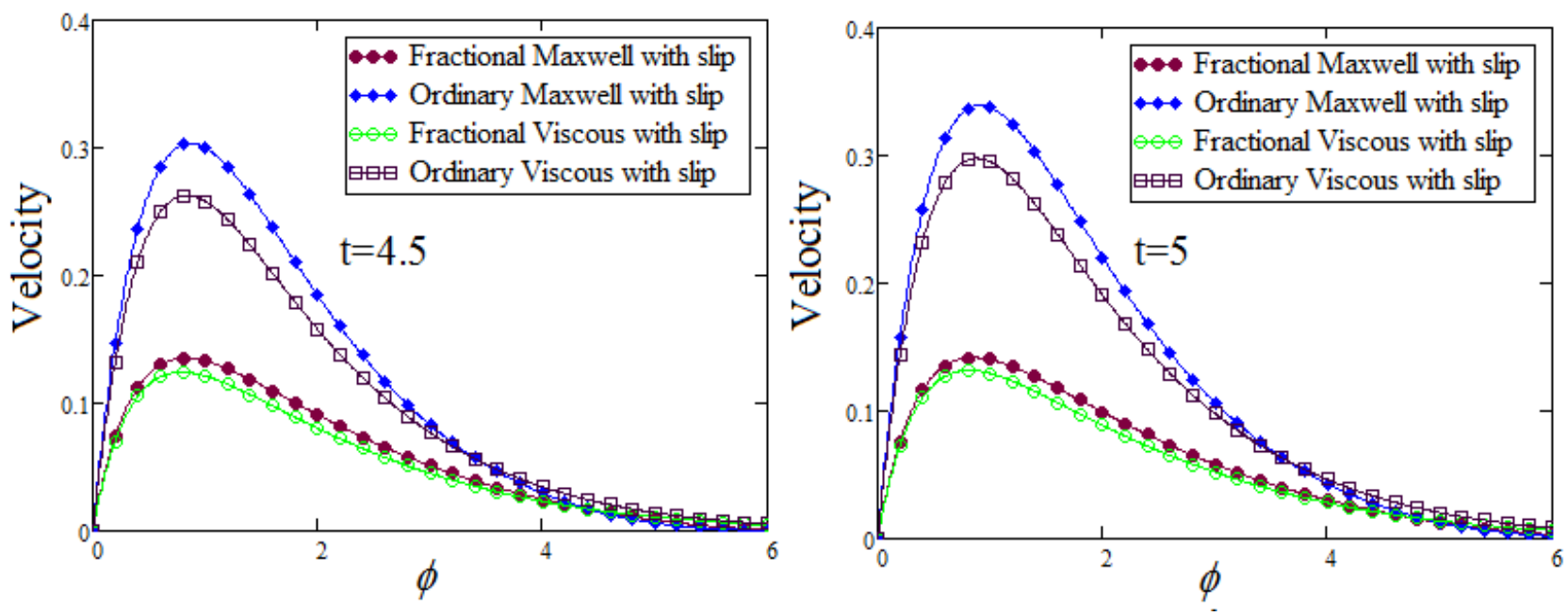

Figure 17. Comparison of velocity profile for the fractional viscous, fractional Maxwell, classical viscous, and classical Maxwell fluids in the presence of slip conditions at two different levels of time, when $\operatorname{Pr}=12, \mathrm{Gr}=5, \wp=0.4, \mathrm{Gm}=3.5, \omega=0.5, \mathrm{Sc}=9, \alpha=0.5 \beta=0.3$, and $\gamma=0.4$.
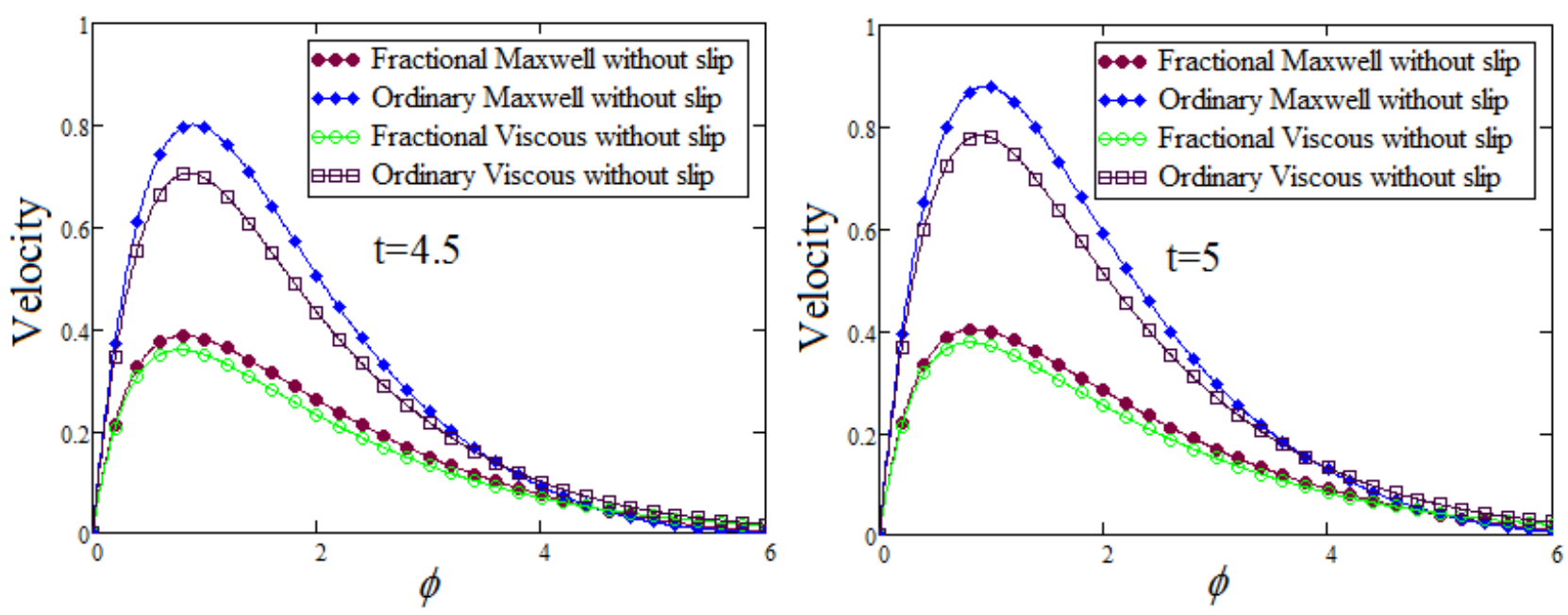

Figure 18. Comparison of velocity profile for the fractional viscous, fractional Maxwell, classical viscous and classical Maxwell fluids in the presence of no slip conditions at two different levels of time, when $\operatorname{Pr}=12, \mathrm{Gr}=5, \mathrm{Gm}=3.5, \omega=0, \wp=0.4, S c=9, \alpha=0.5 \beta=0.3$, and $\gamma=0.4$.

\section{Conclusions}

The prominent feature of this work is to introduce the time dependent, in-compressible, natural convective flow of Maxwell fluid on an infinite, vertical isothermal plate with generalized Mittag-Leffler. For the sake of generalized memory effects, a fractional model was developed by applying the newly introduced Prabhakar fractional operator while having a Mittag-Leffler kernel in the constitutive equations. The work presented in this article is new. A fractionalized diffusion equation is introduced in this model by employing Prabhakar's fractional operator with generalized Fick's law. This Prabhakar-like non integer model has been solved analytically, and exact solutions for dimensionless velocity, concentration, and energy equations are calculated in terms of Mittag-Leffler functions by employing the Laplace transformation technique. The influence of the various system parameters such as $\alpha, P r, \beta, S c, G r, \gamma$, and $G m$ are used to discuss the physical interpretation of the derived results. Some essential findings obtained from graphs are given below:

- It is observed that the temperature profile in cases of slip and no slip conditions decreases when the values of fractional parameters $\alpha, \beta$, and $\gamma$ are elevated. 
- It is seen that temperature and concentration graphs decline corresponding to large values of $P r$ and $S c$, respectively.

- It is detected that, when rising the values of fractional parameters $\alpha, \beta$, and $\gamma$, the concentration profile decreases.

- It is seen that the velocity field in the case of slip and no slip conditions decreases corresponding to elevated the values of fractional parameters.

- The accumulative values of the parameters $S c$ and $P r$ decrease in the velocity field.

- The greater values of the Grashof numbers $G r$ and $G m$ stimulate the velocity contour.

- It is visualized that ordinary Maxwell fluid and ordinary viscous fluid have relatively higher velocity as compared to fractional Maxwell fluid and fractional viscous fluid.

- It is noted that, for classical and fractional models, the velocity field perceived identical behavior for the cases of both slip and zero slip conditions.

- The movement of the fluid in case of zero slip condition is relatively higher as compared to slip conditions.

Author Contributions: Conceptualization, A.U.R. and M.B.R.; Data curation, A.U.R.; Formal analysis, Z.H.S.; Investigation, A.U.R.; Methodology, M.B.R. and Z.H.S.; Project administration, M.B.R.; Software, A.U.R. and Z.H.S.; Validation, M.B.R.; Visualization, Z.H.S.; Writing—original draft, A.U.R. and Z.H.S.; Writing-review \& editing, F.J. and M.B.R. All authors have read and agreed to the published version of the manuscript.

Funding: This research received no external funding.

Institutional Review Board Statement: Not applicable.

Informed Consent Statement: Not applicable.

Data Availability Statement: For the current study, no data sharing is applicable because no data were investigated or developed for this article.

Conflicts of Interest: The authors professed no conflicts of interest.

\section{Nomenclature}

$\begin{array}{lll}\text { Symbol } & \text { Quantity } & \text { Units } \\ \alpha, \beta, \gamma & \text { Fractional parameters } & (-) \\ \mu & \text { Dynamic viscosity } & \left(\mathrm{Kgm}^{-1} \mathrm{~s}^{-1}\right) \\ v & \text { Kinematic coefficient of viscosity } & \left(\mathrm{m}^{2} \mathrm{~s}^{-1}\right) \\ g & \text { Acceleration due to gravity } & \left(\mathrm{m} \cdot \mathrm{s}^{-2}\right) \\ \beta_{1} & \text { Volumetric coefficient of thermal expansion } & \left(\mathrm{K}^{-1}\right) \\ \beta_{2} & \text { Volumetric coefficient of concentration expansion } & \left(\mathrm{K}^{-1}\right) \\ \rho & \text { Fluid density } & \left(\mathrm{Kgm}^{-3}\right) \\ \sigma & \text { Electrical conductivity } & \left(\mathrm{sm}^{-1}\right) \\ C_{p} & \text { Specific heat capacity of fluid } & \left(\mathrm{Jkg}^{-1} \mathrm{~K}^{-1}\right) \\ \xi & \text { Laplace Transform parameter } & (-) \\ Q & \text { Heat generation/absorption } & \left(\mathrm{JK}^{-1} \mathrm{~m}^{-3} \mathrm{~s}^{-1}\right) \\ u & \text { Non-dimensional velocity } & (-) \\ T & \text { Dimensionless temperature } & (-) \\ G_{r} & \text { Thermal Grashof number } & (-) \\ G_{m} & \text { Mass Grashof number } & (-) \\ C_{w} & \text { Concentration of the fluid near the plate } & \left.\mathrm{kgm}^{-3}\right) \\ C_{\infty} & \text { Concentration of the fluid far away from the plate } & \mathrm{kgm}^{-3} \\ T_{w} & \text { Temperature of the plate } & (\mathrm{K}) \\ T_{\infty} & \text { Temperature of fluid far away from the plate } & \left(\mathrm{K}^{-}\right. \\ \lambda & \text { Relaxation time } & (-) \\ D_{m} & \text { Mass diffusivity } & \left(\mathrm{m}^{2} \mathrm{~s}^{-1}\right) \\ u_{0} & \text { Characteristic velocity } & \left(\mathrm{ms}^{-1}\right) \\ & & \end{array}$




$\begin{array}{lll}P_{r} & \text { Prandtl number } & (-) \\ S_{c} & \text { Schmidt number } & (-) \\ B_{0} & \text { Imposed Magnetic field } & \left(\mathrm{Wm}^{-2}\right) \\ M & \text { Total Magnetic field } & (-) \\ k & \text { Thermal conductivity of the fluid } & \left(\mathrm{Wm}^{-2} \mathrm{~K}^{-1}\right) \\ \eta & \text { Time } & (\mathrm{s}) \\ P & \text { Pressure } & \left(\mathrm{N} \mathrm{m}^{-2}\right)\end{array}$

\section{References}

1. Kahshan, M.; Lu, D.; Siddiqui, A.M. A Jeffrey fluid model for a porous-walled channel: Application to flat plate dialyzer. Sci. Rep. 2019, 9, 15879. [CrossRef]

2. Mohebbi, R.; Delouei, A.A.; Jamali, A.; Izadi, M.; Mohamad, A.A. Pore-scale simulation of non-Newtonian power-law fluid flow and forced convection in partially porous media: Thermal lattice Boltzmann method. Physic A 2019, 525, 642-656. [CrossRef]

3. Rehman, A.U.; Riaz, M.B.; Saeed, S.T.; Yao, S. Dynamical Analysis of Radiation and Heat Transfer on MHD Second Grade Fluid. Comput. Model. Eng. Sci. 2021, 129, 689-703. [CrossRef]

4. Riaz, M.B.; Abro, K.A.; Abualnaja, K.M.; Akgül, A.; Rehman, A.U.; Abbas, M.; Hamed, Y.S. Exact solutions involving special functions for unsteady convective flow of magnetohydrodynamic second grade fluid with ramped conditions. Adv. Differ. Equ. 2021, 2021, 408. [CrossRef]

5. Riaz, M.B.; Awrejcewicz, J.; Rehman, A.U. Functional Effects of Permeability on Oldroyd-B Fluid under Magnetization: A Comparison of Slipping and Non-Slipping Solutions. Appl. Sci. 2021, 11, 11477. [CrossRef]

6. Khan, Z.; Tairan, N.; Mashwani, W.K.; Rasheed, H.U.; Shah, H.; Khan, W. MHD and slip effect on two-immiscible third grade fluid on thin film flow over a vertical moving belt. Open Phys. 2019, 17, 575-586. [CrossRef]

7. Rajagopal, K.R.; Gupta, A.S. An exact solution for the flow of a non-Newtonian fluid past an infinite porous plate. Meccanica 1984, 19, 158-161. [CrossRef]

8. Rajagopal, K.R. Mechanics of non-Newtonian fluids in recent development in theoretical fluid Mechanics. Pitman. Res. Notes Math. 1993, 291, 129-162.

9. Erdogan, M.E. On unsteady motions of a second-order fluid over a plane wall. Int. J. Nonlinear. Mech. 2003, 38, 1045-1051. [CrossRef]

10. Labropulu, F. A few more exact solutions of a second grade fluid via inverse method. Mech. Res. Commun. 2000, 27, 713-720. [CrossRef]

11. Fetecau, C.; Fetecau, C.; Rana, M. General solutions for the unsteady flow of second grade fluid over an infinite plate that applies arbitratry shear to the fluid. Z. Naturforsch. 2011, 66, 753-759. [CrossRef]

12. Tawari, A.K.; Ravi, S.K. Analytical studies on transient rotating flow of a second grade fluid in a porous medium. Adv. Theor. Appl. Mech. 2009, 2, 23-41.

13. Islam, S.; Bano, Z.; Haroon, T.; Siddiqui, A.M. Unsteady poiseuille flow of second grade fluid in a tube of elliptical cross section. Proc. Rom. Acad. A 2011, 12, 291-295.

14. Maxwell, J.C. On the dynamical theory of gases. Philos. Trans. Roy. Soc. Lond. 1867, 157, 49-88.

15. Olsson, F.; yström, J. Some properties of the upper convicted Maxwell model for viscoelastic fluid flow. J. Non-Newtonian Fluid Mech. 1993, 48, 125-145. [CrossRef]

16. Aman, S.; Al-Mdallal, Q.; Khan, I. Heat transfer and second order slip effect on MHD flow of fractional Maxwell fluid in a porous medium. J. King Saud Univ. 2020, 32, 450-458. [CrossRef]

17. Choi, J.J.; Rusak, Z.; Tichy, J.A. Maxwell fluid suction flow in a channel. J. Non-Newtonian Fluid Mech. 1999, 85, 165-187. [CrossRef]

18. Khan, M.; Malik, M.Y.; Salahuddin, T.; Saleem, S.; Hussain, A. Change in viscosity of Maxwell fluid flow due to thermal and solutal stratifications. J. Mol. Liq. 2019, 288, 110970. [CrossRef]

19. Fetecau, C.; Fetecau, C. A new exact solution for the flow of a Maxwell fluid past an infinite plate. Int. J. Non Lin. Mech. 2003, 38, 423-427. [CrossRef]

20. Fetecau, C.; Fetecau, C. The Rayleigh Stokes problem for a fluid of Maxwellian type. Int. J. Non Lin. Mech. 2003, 38, 603-607. [CrossRef]

21. Hosseinzadeh, K.; Gholinia, M.; Jafari, B.; Ghanbarpour, A.; Olfian, H.; Ganji, D.D. Nonlinear thermal radiation and chemical reaction effects on Maxwell fluid flow with convectively heated plate in a porous medium. Heat Transf. Asian Res. 2019, 48, 744-759. [CrossRef]

22. Riaz, M.B.; Awrejcewicz, J.; Rehman, A.U.; Abbas, M. Special functions-based solutions of unsteady convective flow of a MHD Maxwell fluid for ramped wall temperature and velocity with concentration. Adv. Differ. Equ. 2021, 2021, 500. [CrossRef]

23. Riaz, M.B.; Rehman, A.U.; Awrejcewicz, J.; Akgül, A. Power Law Kernel Analysis of MHD Maxwell Fluid with Ramped Boundary Conditions: Transport Phenomena Solutions Based on Special Functions. Fractal Fract. 2021, 5, 248. [CrossRef]

24. Hammouch, Z.; Yavuz, M.; Özdemir, N. Numerical solutions and synchronization of a variable-order fractional chaotic system. Math. Model. Numer. Simul. Appl. 2021, 1, 11-23. [CrossRef] 
25. Veeresha, P.; Yavuz, M.; Baishya, C. A computational approach for shallow water forced Korteweg-De Vries equation on critical flow over a hole with three fractional operators. Int. J. Optim. Control. Theor. Appl. (IJOCTA) 2021, 11, 52-67. [CrossRef]

26. Bonyah, E.; Yavuz, M.; Baleanu, D.; Kumar, S. A robust study on the listeriosis disease by adopting fractal-fractional operators. Alex. Eng. J. 2022, 61, 2016-2028. [CrossRef]

27. Yavuz, M.; Sene, N. Stability analysis and numerical computation of the fractional predator-prey model with the harvesting rate. Fractal Fract. 2020, 4, 35. [CrossRef]

28. Riaz, M.B.; Awrejcewicz, J.; Rehman, A.U.; Akgül, A. Thermophysical Investigation of Oldroyd-B Fluid with Functional Effects of Permeability: Memory Effect Study Using Non-Singular Kernel Derivative Approach. Fractal Fract. 2021, 5, 124. [CrossRef]

29. Atangana, A.; Baleanu, D. New fractional derivative with non local and non-singular kernel: Theory and application to heat transfer model. Therm. Sci. 2016, 20, 763-769. [CrossRef]

30. Rehman, A.U.; Shah, Z.H.; Riaz, M.B. Application of Local and Non-local Kernels: The Optimal Solutions of Water-based Nanoparticles Under Ramped Conditions. Progr. Fract. Differ. Appl. 2021, 7, 317-335. http://dx.doi.org/10.18576/pfda/070410.

31. Yavuz, M.; Sene, N. Approximate Solutions of the Model Describing Fluid Flow Using Generalized $\rho$-Laplace Transform Method and Heat Balance Integral Method. Axioms 2020, 9, 123. [CrossRef]

32. Giusti, A.; Colombaro, I. Prabhakar-like fractional viscoelasticity. Commun. Nonlinear Sci. Numer. Simulat. 2018, 56, 138-143. [CrossRef]

33. Riaz, M.B.; Iftikhar, N. A comparative study of heat transfer analysis of MHD Maxwell fluid in view of local and non-local differential operators. Chaos Solitons Fractals 2020, 132, 109556. [CrossRef]

34. Ozkose, F.; Yılmaz, S.; Yavuz, M.; Öztürk, İ; Şenel, M.T.; Bağcl, B.Ş; Doğan, M.; Önal, Ö. A Fractional Modeling of Tumor-Immune System Interaction Related to Lung Cancer with Real Data. Eur. Phys. J. Plus 2022, 137, 1-28. [CrossRef]

35. Ozkose, F.; Yavuz, M. Investigation of interactions between COVID-19 and diabetes with hereditary traits using real data: A case study in Turkey. Comput. Biol. Med. 2021, 141, 105044. [CrossRef]

36. Naik, P.A.; Yavuz, M.; Qureshi, S.; Zu, J.; Townley, S. Modeling and analysis of COVID-19 epidemics with treatment in fractional derivatives using real data from Pakistan. Eur. Phys. J. Plus 2020, 135, 1-42. [CrossRef] [PubMed]

37. Ikram, R.; Khan, A.; Zahri, M.; Saeed, A.; Yavuz, M.; Kumam, P. Extinction and stationary distribution of a stochastic COVID-19 epidemic model with time-delay. Comput. Biol. Med. 2022, 141, 105115. [CrossRef] [PubMed]

38. Allegretti, S.; Bulai, I.M.; Marino, R.; Menandro, M.A.; Parisi, K. Vaccination effect conjoint to fraction of avoided contacts for a Sars-Cov-2 mathematical model. Math. Model. Numer. Simul. Appl. 2021, 1, 56-66. [CrossRef]

39. Joshi, H.; Jha, B.K. Chaos of calcium diffusion in Parkinson's infectious disease model and treatment mechanism via Hilfer fractional derivative. Math. Model. Numer. Simul. Appl. 2021, 1, 84-94.

40. Rehman, A.U.; Riaz, M.B.; Awrejcewicz, J.; Baleanu, D. Exact solutions of thermomagetized unsteady non-singularized jeffery fluid: Effects of ramped velocity,concentration with newtonian heating. Results Phys. 2021, 26, 104367. [CrossRef]

41. Rehman, A.U.; Riaz, M.B.; Akgul, A.; Saeed, S.T.; Baleanu, D. Heat and mass transport impact on MHD second grade fluid: A comparative analysis of fractional operators. Heat Transf. 2021, 50, 7042-7064. [CrossRef]

42. Rehman, A.U.; Riaz, M.B.; Rehman, W.; Awrejcewicz, J.; Baleanu, D. Fractional Modeling of Viscous Fluid over a Moveable Inclined Plate Subject to Exponential Heating with Singular and Non-Singular Kernels. Math. Comput. Appl. 2022, 27, 8. [CrossRef]

43. Riaz, M.B.; Rehman, A.U.; Awrejcewicz, J.; Jarad, F. Double Diffusive Magneto-Free-Convection Flow of Oldroyd-B Fluid over a Vertical Plate with Heat and Mass Flux. Symmetry 2022, 14, 209. [CrossRef]

44. Zhang, X.-H.; Shah, R.; Saleem, S.; Shah, N.A.; Khan, Z.A.; Chung, J.D. Natural convection flow maxwell fluids with generalized thermal transport and newtonian heating. Case Stud. Therm. Eng. 2021, 27, 101226. [CrossRef]

45. Shah, N.A.; Fetecau, C.; Vieru, D. Natural convection flows of Prabhakar-like fractional Maxwell fluids with generalized thermal transport. J. Therm. Anal. Calorim. 2021, 143, 2245-2258. [CrossRef] 